\title{
Contests with rank-order spillovers
}

\author{
Michael R. Baye · Dan Kovenock • \\ Casper G. de Vries
}

Received: 9 September 2008 / Accepted: 29 June 2009 / Published online: 28 August 2009 (C) The Author(s) 2009. This article is published with open access at Springerlink.com

\begin{abstract}
This paper presents a unified framework for characterizing symmetric equilibrium in simultaneous move, two-player, rank-order contests with complete information, in which each player's strategy generates direct or indirect affine "spillover" effects that depend on the rank-order of her decision variable. These effects arise in natural interpretations of a number of important economic environments, as well as in classic contests adapted to recent experimental and behavioral models where individuals exhibit inequality aversion or regret. We provide the closed-form solution for the symmetric Nash equilibria of this class of games, and show how it can be used to directly solve for equilibrium behavior in auctions, pricing games, tournaments, $\mathrm{R} \& \mathrm{D}$ races, models of litigation, and a host of other contests.
\end{abstract}

\footnotetext{
We are grateful to two referees for exceptionally lucid and helpful reports. We have also benefited from conversations with Murali Agastya, Chaim Fershtman, Srihari Govindan, Rick Harbaugh, Wolfgang Leininger, Benny Moldovanu, and Michael Rauh. Finally, we thank Nels Leader, participants in the conference on "Contests: Theory and Applications" at the Stockholm School of Economics, and participants at the 2008 CESifo Workshop on "Advances in the Theory of Contests and its Applications" in Venice. Kovenock has benefited from the financial support of the Wissenschaftszentrum Berlin für Sozialforschung.
}

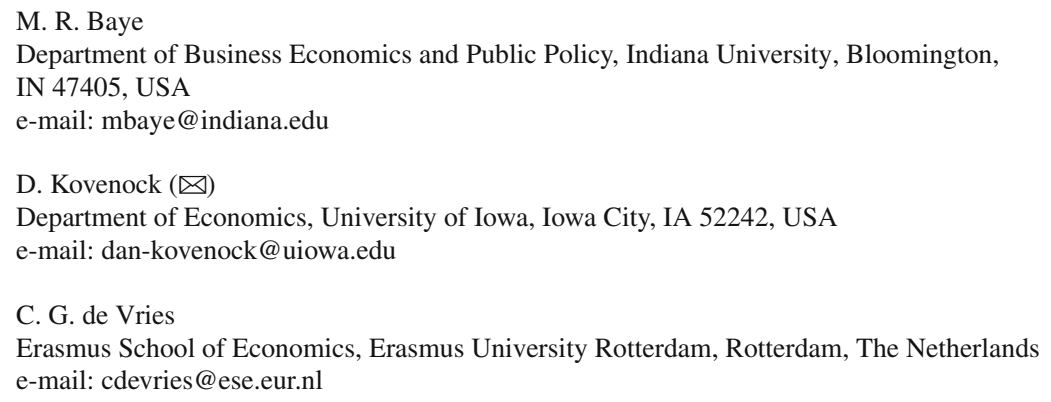


Keywords Contests $\cdot$ Auctions $\cdot$ Spillovers

JEL Classification $\mathrm{C} 72 \cdot \mathrm{C} 73 \cdot \mathrm{D} 43 \cdot \mathrm{D} 44 \cdot \mathrm{D} 74$

\section{Introduction}

This paper presents a unified framework for analyzing equilibrium in simultaneousmove, two-player, rank-order contests with complete information, in which each player's strategy generates direct or indirect affine "spillover" effects that depend on the rank-order of her decision variable. We show that these effects arise in natural interpretations of a number of important economic environments, including contests adapted to recent experimental and behavioral models where individuals exhibit inequality aversion or regret. We provide a characterization of symmetric equilibria (both pure and mixed), closed-form expressions for these equilibria, and show how our results may be used to directly solve for equilibrium behavior in auctions, pricing games, tournaments, R\&D races, models of litigation, and a host of other games.

Rank-order contests are ubiquitous. These take the form of environments in which players choose non-negative bids (which may be interpreted as a proposed payment, effort, or the commitment of other scarce resources that are non-refundable) whose rank-order discontinuously influences the probability of winning some prize. Classic examples include homogeneous product Bertrand competition (see Bertrand 1883), in which the lowest price firm "wins" the profit from selling to demand at that price, as well as first- and second-price auctions (see Vickrey 1961), where the player who submits the highest bid wins the item and pays either his own bid (in the first-price auction) or the bid of the second-highest bidder (in the second-price auction).

Winners and losers alike forfeit payments in many rank-order contests. In a firstprice all-pay auction, for instance, each player submits a non-refundable bid and only the higher bidder receives a prize. The war-of-attrition (see Maynard Smith 1974) is a second-price all-pay auction: the high bidder wins the prize and pays the amount bid by the second-highest bidder. These forms of competition have been widely used to model activities as diverse as patent and R\&D races, lobbying and rent-seeking activities, litigation, advertising and political campaigns, tournaments as incentive devices in labor markets, competition for college admissions, sports competitions, urban architecture, and territorial contests among organisms. ${ }^{1}$

The principal motivation of this article is that spillovers are often important in rank-order contests; in many economic environments, one player's decision affects the other player's payoff, and the nature of this effect may depend on the rank-order of the players' choices. This is perhaps most obvious in second-price auctions where the high bidder pays the second-highest bid, but spillovers also arise in a variety of economic contexts. For instance, an extensive literature starting with D'Aspremont and Jacquemin (1988) has examined the effects of positive spillovers in R\&D competition

\footnotetext{
1 Applications in these areas include work by Dasgupta (1986), Kaplan et al. (2003), Hillman and Riley (1989), Baye et al. (1993), Che and Gale (1998), Baye et al. (2005), Sahuguet and Persico (2006), Konrad (2004), Fu (2006), Groh et al. (2009), Helsey and Strange (2008), and Kura (1999).
} 
that can arise when one player's R\&D effort provides information that benefits its rival. Although D'Aspremont and Jacquemin (1988) does not involve rank-order effects, a growing literature, starting from an original observation by Dasgupta (1986), models the R\&D process as a rank-order tournament (see also Che and Gale 2003; Zhou 2006). The results examined in this article apply to the positive spillovers arising in this context.

Rank-order spillovers also arise in models of litigation. Baye et al. (2005), for instance, examine equilibrium in a litigation game with incomplete information in which legal expenditures increase the quality of the case presented and where the "best case" wins. ${ }^{2}$ This turns the litigation process into a rank-order contest in which the litigation incentives in legal systems, such as the American, British, Continental and "Quayle" systems, may be examined. Although the American system, where litigants pay their own legal costs, involves no spillovers, other fee-shifting rules, such as the British and Continental rules, which require that losers compensate winners for a portion of their legal costs, and the Quayle system, in which the loser reimburses the winner up to the amount actually spent by the loser, involve spillovers. Under the British and Continental rules there is a negative indirect spillover effect of the winner's expenditure on the loser. In the continuation we call this a second-order negative spillover effect. In the case of the Quayle system, there is a positive indirect spillover of the loser's expenditure on the winner. We call this a first-order positive spillover effect.

Our taxonomy of spillover effects may also be used to construct and analyze variants and extensions of the auction and contest literatures noted above. For instance, the classic partnership dissolution problem may be viewed as the auction of a business in which two partners simultaneously submit bids and the partner with the higher bid pays his bid to the partner with the lower bid in return for ownership of the business. In this case, the payment of the winning partner is a second-order positive spillover effect on the loser. Similarly, both the first-price and second-price all-pay auctions, often used to model economic and biological contests, may be extended to include environments in which effort expended imposes both a rank-order contingent direct effect on the player expending the effort and a rank-order spillover effect on the player's rival. For instance, if two organisms are engaged in a territorial fight, the effort of the winner may exact both a cost to the winner (a first-order negative direct effect) and a cost to the loser (a second-order negative spillover effect). The loser's effort may have a second-order negative direct effect on the loser's payoff and a first-order negative spillover effect on the winner.

An important class of economic environments where rank-order dependent spillovers arise naturally is the analysis of auctions adapted to recent experimental and behavioral models of individual choice. In Sect. 3 we show that our characterization may be used to examine behavioral models that include: (i) tournaments in which

\footnotetext{
2 Our analysis is also related to a number of papers that have examined other incomplete information environments. Recent contributions include the analysis of cross-holdings and financial externalities (Dasgupta and Tsui 2004; Ettinger 2003; Maasland and Onderstal 2007), k-double auctions (Kittsteiner 2003); and charity auctions (Engers and McManus 2004; Goeree et al. 2005). Our paper is also related to the literature on auctions with externalities dependent on the identity of the winner and not the bids per se (see, for instance, Jehiel et al. 1996).
} 
individuals exhibit inequality aversion in the spirit of Fehr and Schmidt (1999), (ii) first-price and all-pay auctions where players experience regret similar to that in the models of Engelbrecht-Wiggans (1989), Engelbrecht-Wiggans and Katok (2007), and Filiz-Ozbay and Ozbay (2007), and (iii) an all-pay auction in which players maximize relative fitness according to the finite agent Evolutionary Stable Strategies (ESS) equilibrium of Schaffer (1988). ${ }^{3}$

Section 3 also shows that many pricing games have rank-order dependent spillovers that may be analyzed within our framework. For instance, in a variant of the classic Bertrand model due to Varian (1980), two sellers simultaneously set a price and sell to three inelastic segments of demand with common choke price, $r$. One of these inelastic segments consists of price-sensitive consumers who are aware of both prices in the market and who purchase from the lower-price seller, while the other two segments are attached to different firms and are each aware of only the price of that firm to which they are attached (as long as that price is at or below the choke price). Baye et al. (1992) have shown that this game has a structure similar to that of a first-price all-pay auction in which the bid is the difference between the choke price and a firm's price. In this context, the bid corresponds to the opportunity cost of the lost revenue from the seller's own uninformed segment that results from reducing price in order to attempt to capture the "prize" consisting of the demand of the informed price-sensitive consumer segment.

Spillovers also arise naturally in the context of the Varian model when one examines popular price matching policies (see Lin 1988; Png and Hirshleifer 1987; Baye and Kovenock 1994). If a high-price seller institutes a price-matching policy, it will sell at its own price to consumers informed only of that price, but sell at its rival's price to a proportion of the informed customers who are willing to bear the cost of visiting the high-price seller and taking it up on its offer to match the better price. In this case, the rival's low price generates a spillover effect on the high-price seller's payoff, but not vice-versa. Section 3 also includes additional applications of our results, including a "reference pricing" version of the Varian model that includes "relative bargain" seekers whose demand from the low-price firm depends on the ratio of the high price to the low price. With reference pricing, a rival's high price generates a spillover effect on the low-price seller's payoff, but not vice-versa.

All of these models have the property that they are special cases of the linear parameterized class of rank-order contests whose symmetric equilibria we characterize in this paper. In Sect. 2 we formally introduce this class of models and provide a general closed-form solution for the symmetric equilibria of the class. We characterize the symmetric equilibrium strategies as functions of "contest parameters," which when varied change the "rules" of the contest. In Sect. 3, we show how this characterization may be used to directly obtain closed-form solutions for symmetric equilibrium strategies in these and other economic environments. In Sect. 4 we conclude. The proofs are collected in the Appendix.

\footnotetext{
3 See also Hehenkamp et al. (2004) who, to the best of our knowledge, were the first to apply the ESS equilibrium concept in a (Tullock) contest.
} 


\section{Model and results}

We study the symmetric Nash equilibria of the class of two-player games of complete information in which each player $i \in\{1,2\}$ chooses an action (or bid) $x_{i}$ from the strategy space $A=[0, \infty)$, and where payoffs are

$$
u_{i}\left(x_{i}, x_{j}\right)= \begin{cases}W\left(x_{i}, x_{j}\right) \equiv v-\beta x_{i}-\delta x_{j} & \text { if } x_{i}>x_{j} \\ L\left(x_{i}, x_{j}\right) \equiv-\gamma-\alpha x_{i}-\theta x_{j} & \text { if } x_{i}<x_{j} \\ T\left(x_{i}, x_{j}\right) \equiv \frac{1}{2} W\left(x_{i}, x_{j}\right)+\frac{1}{2} L\left(x_{i}, x_{j}\right) & \text { if } x_{i}=x_{j}\end{cases}
$$

We assume that $V \equiv v+\gamma>0 .{ }^{4}$ We also assume that at least one of the contest parameters $\beta, \delta, \alpha$, or $\theta$ is nonzero. ${ }^{5}$ In the sequel, we let $\Gamma$ denote an arbitrary game within this class.

The $\delta$ and $\theta$ parameters capture the externalities (negative or positive) that contestants may inflict on each other. We use the terms "first-order positive (negative) spillover effects" when $\delta<(>) 0$, and "second-order positive (negative) spillover effects" when $\theta<(>) 0$. This captures the fact that when $x_{i}$ is the higher bid (the first in rank-order), the spillover effect of player $j$ 's bid, $j \neq i$, on player $i$ 's payoff is linear with coefficient $-\delta$. If $\delta>0$, this effect is negative and if $\delta<0$ this effect is positive. Likewise, when player $i$ 's bid is the lower bid (second in the rank-order), the spillover effect of player $j$ 's bid, $j \neq i$, is linear with coefficient $-\theta$. If $\theta>0$, this effect is negative and if $\theta<0$ this effect is positive. For similar reasons, we refer to $\beta$ and $\alpha$ as the first- and second-order direct effects. If player $i$ 's bid $\left(x_{i}\right)$ is the higher bid, or first, in the rank-order, the direct effect of player $i$ 's bid on player $i$ 's payoff is linear with coefficient $-\beta$. If $\beta>0$, the first-order direct effect of an increase in player $i$ 's bid is negative; this effect is positive if $\beta<0$. Similar interpretations apply to the second-order direct effect, $\alpha$.

Notice that, were the strategy space bounded and one is merely interested in establishing existence of a symmetric equilibrium, one could readily analyze this class of games using the Dasgupta and Maskin (1986) framework. For games of incomplete information, Lizzeri and Persico (2000) examine existence and uniqueness of bidding strategies in auctions where $W\left(x_{i}, x_{j}\right)$ and $L\left(x_{i}, x_{j}\right)$ need not be linear, while Baye et al. (2005) provide closed-form expressions for equilibrium strategies in the linear case. In what follows, we provide closed-form solutions for symmetric pure- and mixed-strategy equilibria for the case of complete information, and allow for parameter configurations not accounted for in the Lizzeri-Persico and Baye-Kovenock-deVries analyses. ${ }^{6}$

\footnotetext{
${ }^{4}$ In particular, note that $V>0$ implies $W(0,0)>L(0,0)$.

5 The case where $\beta=\delta=\alpha=\theta=0$ corresponds to the game of "pick the greatest non-negative real number," which does not have an equilibrium when the strategy space is unbounded.

${ }^{6}$ Complete information analogs of the Lizzeri-Persico axioms (labelled A1-A8) would require $\alpha \geq 0$ (A5, A7), $\beta>0$ (A7), $\delta \geq 0$ (A7), and $\theta=0$ (A7). Baye, Kovenock, and de Vries assume, in their incomplete information framework, that $(\beta, \alpha)>0, \delta=(1-\alpha)$, and $\theta=(1-\beta)$.
} 


\subsection{Symmetric pure strategy Nash equilibria}

We first provide the conditions under which there exists a symmetric pure strategy equilibrium, $x^{*}$, such that each player earns the equilibrium payoff $U^{*}<\infty$. To this end, define $\eta \equiv \alpha+\theta-\beta-\delta$, so that $\eta$ measures the change in the payoff per unit of expenditure at $x^{*}$ derived solely from changes in the direct and spillover effects (and not from winning $V$ ) that result from the switch from being a loser to being a winner at $x^{*}$.

Proposition $1 \Gamma$ has a symmetric pure strategy Nash equilibrium if and only if the following three conditions jointly hold: (i) $\beta \geq 0$, (ii) $\alpha \leq 0$ and (iii) $\eta<0$. Furthermore, there is but one such equilibrium and it is given by

$$
x^{*}=-\frac{V}{\eta} \equiv \frac{v+\gamma}{\beta+\delta-\alpha-\theta} .
$$

These conditions have intuitive interpretations: $\beta \geq 0$ means that, conditional on winning, a player's utility is non-increasing in his own bid, and $\alpha \leq 0$ means that, conditional on losing, a player's utility is non-decreasing in his own bid. The condition $\eta<0$ roughly means that in addition, the net spillover effect $(\theta-\delta)$ is "small" relative to the net direct effect $(\alpha-\beta)$.

Examples of applications of Proposition 1 to games of complete information include the first-price auction ( $\gamma=0, \beta=1, \delta=\alpha=\theta=0$ ) where $x^{*}=v$ and the second-price auction ( $\gamma=0, \delta=1, \beta=\alpha=\theta=0$ ) where $x^{*}=v$. Proposition 1 also implies that games such as the standard first-price all-pay auction $(\beta=\alpha=1$, $\delta=\theta=0$ ) and the second-price all-pay auction (also called the war of attrition, where $\delta=\alpha=1, \beta=\theta=0$ ) do not have symmetric pure-strategy equilibria. Since it is known that these special cases of $\Gamma$ do have symmetric mixed-strategy equilibria, we next provide a characterization of all such equilibria to $\Gamma$.

\subsection{Symmetric mixed-strategy equilibria}

In the analysis that follows, a cumulative distribution function, $F$, is said to be a symmetric mixed-strategy equilibrium of $\Gamma$ (with associated equilibrium payoff $E U^{*}$ ) if, for each player $i$, for every $x_{i}$ in the support of $F$, and for all $x_{i}^{\prime} \in[0, \infty)$ :

$$
\begin{aligned}
U_{i}\left(x_{i}^{\prime}, F\right) & \equiv \int_{0}^{\infty} u_{i}\left(x_{i}^{\prime}, x_{j}\right) \mathrm{d} F\left(x_{j}\right) \leq \int_{0}^{\infty} u_{i}\left(x_{i}, x_{j}\right) \mathrm{d} F\left(x_{j}\right) \equiv U_{i}\left(x_{i}, F\right) \\
& =E U^{*} \in(-\infty, \infty) .
\end{aligned}
$$

Note that the existence of such an equilibrium requires that, for each $x_{i} \in[0, \infty), u_{i}\left(x_{i}\right.$, $x_{j}$ ) be integrable with respect to the probability measure that induces $F\left(x_{j}\right)$; that is, conditional expected utility exists and is finite. ${ }^{7}$ This formulation of symmetric mixed-

\footnotetext{
7 See, for instance, Chung (1974, p. 40) for a formal definition of integrability.
} 
strategy equilibria permits us to deal with technical issues that can arise in games with unbounded strategy spaces and payoffs. ${ }^{8}$

It follows that if $F$ is a symmetric mixed-strategy equilibrium with density $f(x)$ on some subset $(m, u)$ of the support of $F$ (so that there are no mass points in this interval), the expected payoff to a player that bids $w \in(m, u)$ against the rival's mixed-strategy $F$ is given by

$$
E U(w)=\int_{0}^{w} W(w, x) \mathrm{d} F(x)+\int_{w}^{\infty} L(w, x) \mathrm{d} F(x) .
$$

Since $F$ is a symmetric mixed-strategy equilibrium by hypothesis, $E U(w)=E U^{*}$ on $w \in(m, u)$. Hence,

$$
\frac{\mathrm{d} E U(w)}{\mathrm{d} w}=[V+\eta w] f(w)-\alpha+(\alpha-\beta) F(w)=0
$$

on $(m, u)$. The solution to this differential equation is given by

$$
F(w)=\frac{\alpha}{\alpha-\beta}\left\{1-\left[\frac{V+\eta m}{V+\eta w}\right]^{\frac{\alpha-\beta}{\eta}}\right\}+C\left[\frac{V+\eta m}{V+\eta w}\right]^{\frac{\alpha-\beta}{\eta}},
$$

where $m \geq 0$ and $0 \leq C \leq 1$.

Notice that this derivation of the functional form for a symmetric equilibrium mixed-strategy is only heuristic, as it ignores mass points, the possibility of profitable deviations outside of $(m, u)$, and furthermore, may not represent a well-defined distribution function for some parameter configurations. Our next proposition addresses these issues formally and characterizes the non-degenerate symmetric mixed-strategy equilibria to $\Gamma$.

Proposition $2 \Gamma$ has a non-degenerate symmetric mixed-strategy equilibrium if and only if one of the following three sets of conditions holds: (i) $\beta>0$ and $\alpha>0$; (ii) $\beta=0, \alpha>0$ and either $\eta \theta=0$ or $\eta<\alpha$; or (iii) $\beta=0, \alpha<0$ and either $\alpha<\eta<0$ or $\eta<\theta=0$. In cases (i) and (ii), the equilibrium is unique within the class of symmetric equilibria (pure or mixed). In case (iii) there exists a continuum of non-degenerate symmetric mixed-strategy equilibria, as well as a unique symmetric pure strategy equilibrium (given in Proposition 1). The non-degenerate symmetric mixed-strategy equilibria are atomless and described by the distribution function $F^{*}(w)$ on $\left[m^{*}, u^{*}\right)$, where

\footnotetext{
8 The fact that $F$ satisfies the above definition of an equilibrium does not imply that $u_{i}\left(x_{i}, x_{j}\right)$ is integrable with respect to the the joint probability measure induced by $F$ because $E\left[u_{i}\left(x_{i}, x_{j}\right)\right]$ may not exist. Note that if $E\left[u_{i}\left(x_{i}, x_{j}\right)\right]$ exists, it equals $E U^{*}$; if it does not exist, $F$ is nonetheless an equilibrium in the sense that any given $x_{i}$ drawn from $F$ and assigned to player $i$ is a best response to $F$-provided player $i$ knows this realization, but not the realization assigned to his rival.
} 


$$
\begin{aligned}
& F^{*}(w)= \begin{cases}\frac{\alpha}{\alpha-\beta}\left(1-\left(\frac{V+\eta m^{*}}{V+\eta w}\right)^{\frac{\alpha-\beta}{\eta}}\right) & \text { if } \eta \neq 0 ; \alpha \neq \beta \\
\frac{\alpha}{\theta-\delta} \ln \left(\frac{V+(\theta-\delta) w}{V}\right) & \text { if } \eta \neq 0 ; \alpha=\beta \\
\frac{\alpha}{\alpha-\beta}\left(1-\exp \left(-\frac{\alpha-\beta}{V} w\right)\right) & \text { if } \eta=0 ; \alpha \neq \beta \\
\frac{\alpha}{V} w & \text { if } \eta=0 ; \alpha=\beta\end{cases} \\
& m^{*}=\left\{\begin{array}{ll}
0 & \text { if } \alpha>0 \\
m^{\prime} \in\left(-\frac{V}{\eta}, \infty\right) & \text { if } \alpha<0
\end{array}\right. \text {, and } \\
& u^{*}= \begin{cases}-\frac{V}{\eta} & \text { if } \alpha>0 ; \beta=0 ; \eta<0 \\
\frac{V}{\eta}\left[(\alpha / \beta)^{\frac{\eta}{\alpha-\beta}}-1\right] & \text { if } \alpha>0 ; \beta>0 ; \alpha \neq \beta ; \eta \neq 0 \\
\frac{V}{\eta}[\exp (\eta / \alpha)-1] & \text { if } \alpha=\beta>0 ; \eta \neq 0 \\
\frac{V}{\alpha-\beta} \ln \frac{\alpha}{\beta} & \text { if } \alpha>0 ; \beta>0 ; \alpha \neq \beta ; \eta=0 \\
V / \alpha & \text { if } \alpha>0 ; \beta>0 ; \alpha=\beta ; \eta=0 \\
\infty & \text { if otherwise. }\end{cases}
\end{aligned}
$$

Furthermore, the corresponding equilibrium payoffs are given by

$$
E U^{*}= \begin{cases}\frac{\theta v+\delta \gamma}{\theta-\delta}+\beta \frac{\theta}{\eta(\theta-\delta)}\left[1-\left(\frac{\alpha}{\beta}\right)^{\frac{\eta}{\alpha-\beta}}\right] V & \text { if } \eta \neq 0 ; \alpha \neq \beta ; \theta \neq \delta ; \beta \neq 0 \\ -\gamma+\frac{\theta V}{\eta}-\frac{\theta V}{\eta} \frac{\alpha}{\eta} \ln \frac{\alpha}{\beta} & \text { if } \eta \neq 0 ; \alpha \neq \beta ; \theta=\delta ; \beta \neq 0 \\ \frac{\theta v+\delta \gamma}{\theta-\delta}+\frac{\alpha \delta}{\theta-\delta} m^{*} & \text { if } \eta \neq 0 ; \alpha \neq \beta ; \theta \neq 0 ; \beta=0 \\ -\gamma-\alpha m^{*} & \text { if } \eta \neq 0 ; \alpha \neq \beta ; \theta=0 ; \beta=0 \\ \frac{\theta v+\delta \gamma}{\theta-\delta}+\beta \frac{\theta}{(\theta-\delta)^{2}}\left[1-\exp \left(\frac{\theta-\delta}{\beta}\right)\right] V & \text { if } \eta \neq 0 ; \alpha=\beta \\ \frac{\theta v+\delta \gamma}{\theta-\delta}+\beta \frac{\theta}{(\theta-\delta)^{2}}\left[\ln \left(\frac{\alpha}{\beta}\right)\right] V & \text { if } \eta=0 ; \alpha \neq \beta ; \beta \neq 0 \\ \frac{\theta v+\delta \gamma}{\theta-\delta} & \text { if } \eta=0 ; \alpha \neq \beta ; \beta=0 \\ -\gamma-\frac{\theta}{2 \alpha} V & \text { if } \eta=0 ; \alpha=\beta .\end{cases}
$$

Notice that all of the terms in Eqs. (5), (6) and (7) are well-defined, since conditions (i) through (iii), which guarantee the existence of a non-degenerate symmetric mixed-strategy equilibrium, imply: (a) $\alpha \neq 0$; (b) if $\alpha<0$, then $\eta<0$ and $\beta=0$; (c) if $\alpha=\beta$, then $\alpha>0$ and $\beta>0$; (d) if $\alpha=\beta$ and $\eta \neq 0$, then $\theta \neq \delta$; (e) if $\alpha \neq \beta$ and $\eta=0$, then $\theta \neq \delta$; and (f) if $\beta \neq 0$ then $\alpha>0$ and $\beta>0$.

The Appendix constructively derives all of the possible symmetric equilibria and the resulting payoffs in a series of lemmata, and also indicates when an equilibrium does not exist. The analysis in the Appendix implies that one may also obtain closedform expressions for the equilibrium strategies by taking limits of Eq. (4). For instance, 
the functional form for the equilibrium distribution function in Proposition 2 when $\eta \neq 0$ and $\alpha=\beta$ obtains by taking the limit of equation (4) as $\alpha-\beta$ tends to zero.

Propositions 1 and 2, which characterize the parameter ranges where symmetric pure and non-degenerate mixed-strategy equilibria arise, together facilitate a complete partition of the parameter space into ranges of qualitatively different symmetric Nash equilibrium correspondences. We summarize this in

Proposition 3 The symmetric equilibria to $\Gamma$ are characterized as follows:

(a) The unique symmetric equilibrium is in pure strategies if and only if one of the following three conditions holds: (i) $\beta>0, \alpha \leq 0$, and $\eta<0$; (ii) $\beta=0, \alpha=0$, and $\eta<0$; or (iii) $\beta=0, \eta \leq \alpha<0$, and $\theta \neq 0$.

(b) The unique symmetric equilibrium is in non-degenerate mixed strategies if and only if one of the following two conditions holds: (i) $\beta>0$ and $\alpha>0$; or (ii) $\beta=0, \alpha>0$ and either $\eta \theta=0$ or $\eta<\alpha$.

(c) There is a unique symmetric pure strategy equilibrium and a continuum of nondegenerate symmetric mixed-strategy equilibria if and only if $\beta=0, \alpha<0$ and either $\alpha<\eta<0$ or $\eta<\theta=0$.

Furthermore, if none of the conditions in (a) through (c) hold, $\Gamma$ does not have a symmetric equilibrium (either pure or mixed).

It is important to emphasize that we have focused solely on symmetric equilibria. Asymmetric equilibria are known to exist, for instance, in the symmetric two player war of attrition $(\alpha=\delta=1, \beta=\theta=0)$ and sad loser auction $(\alpha=1, \beta=\theta=\delta=0)$. We also note that, were there a common finite upper bound on the strategy space for the players (such that the strategy space is compact), existence of a symmetric equilibrium is guaranteed by Lemma 7 of Dasgupta and Maskin (1986). ${ }^{9}$

Before proceeding, we offer several observations about the four functional forms for the symmetric equilibrium mixed-strategies that can arise under different parameter configurations. First, note that the lower bound of the support, $m^{*}$, of every symmetric equilibrium mixed-strategy to $\Gamma$ is zero when $\alpha>0$, but an arbitrary positive number $m^{*} \in(-V / \eta, \infty)$ when $\alpha<0$ (this accounts for the continuum of symmetric mixed-strategy equilibria that arise in this case). Second, notice that the equilibrium mixed-strategies take on different functional forms (including the uniform distribution, exponential distribution, as well as more exotic forms) depending on which of four regions (denoted R1-R4) the parameters lie:

R1: $\eta \neq 0$ and $\alpha \neq \beta$. In this case, any of the conditions (i), (ii), or (iii) in Proposition 2 may apply. This is, in a sense, the most general form of an all pay auction in which direct effects are asymmetric and spillovers may be either symmetric or asymmetric. When both $\alpha$ and $\beta$ are positive, Proposition 2 implies that the support of the distribution is bounded. When $\beta=0$, the support of the symmetric equilibrium mixed strategies is unbounded unless $\eta<0$; the unbounded distribution is known as a Burr distribution with a Pareto-type upper tail such that not

\footnotetext{
9 As a referee noted, extending our analysis to include a common finite upper bound on the strategy space would permit one to analyze games (such as the Traveler's Dilemma; see Basu 1994) that have payoffs as in Eq. (1) but are not covered in our framework because of the strategy space restriction.
} 
all moments exist. Notice that part (ii) of Proposition 2 includes the case where $\beta=0, \alpha>0$, and $\theta=0$. The Riley and Samuelson (1981) sad loser auction in which only the loser pays his bid $(\beta=\delta=\theta=0$ and $\alpha=1)$ is a special case of this configuration. The case where $\beta=0, \alpha>0$ and $\alpha>\eta \neq 0$ may be viewed as a sad loser auction with spillovers $(\delta>\theta)$. Finally, note that when $\alpha<\beta=0$ and either $\alpha<\eta<0$ or $\eta<\theta=0$, there is a continuum of symmetric mixed-strategy equilibria which stem from the continuum of lower bounds for the support that arise when $\alpha<0$. The Baye and Morgan (1999) folk-theorem for one-shot, homogeneous product Bertrand games, which entails both a continuum of symmetric equilibrium strategies and equilibrium payoffs, is an example of an economic environment that may be viewed as a $\Gamma$ with parameters in R1.

R2: $\eta \neq 0$ and $\alpha=\beta$. In this case, conditions (i) through (iii) in Proposition 2 imply that only condition (i) is satisfied, and therefore $\alpha=\beta>0$ and $\theta \neq \delta$. In this case the asymmetric spillovers impact both the equilibrium payoffs and the symmetric mixed strategies (which take on a logarithmic form). To the best of our knowledge, contests or auctions with parameters in R2 have not heretofore been examined in the literature. Games with parameters in R2 may be interpreted as a generalized first-price all-pay auction with asymmetric spillovers.

R3: $\eta=0$ and $\alpha \neq \beta$. In this case, conditions (i) through (iii) in Proposition 2 imply that either (i) or (ii) applies, and therefore $\alpha>0, \beta \geq 0$ and $\theta \neq \delta$. In this case the asymmetric spillovers impact the symmetric equilibrium payoffs but not the equilibrium mixed-strategies (which take the form of a truncated exponential distribution). The standard war of attrition $(\alpha=\delta=1, \beta=\theta=0)$ is a special case of a $\Gamma$ with parameters in $\mathrm{R} 3$. Notice that when $\beta>0$, the symmetric mixed-strategy has a bounded upper support, whereas it is unbounded when $\beta=0$ (and hence the symmetric mixed-strategies are a non-truncated exponential distribution).

R4: $\eta=0$ and $\alpha=\beta$. In this case, conditions (i) through (iii) in Proposition 2 imply that only condition (i) can be satisfied, and therefore $\alpha=\beta>0$ and $\theta=\delta$. The standard all-pay auction $(\alpha=\beta=1$ and $\theta=\delta=0$ ) is a special case of this configuration. More generally, this configuration is a modified first-price all-pay auction in which $\theta=\delta \neq 0$ is a "nuisance parameter" that does not influence the symmetric equilibrium mixed-strategy (which is a uniform distribution) but does impact the players' equilibrium expected payoffs. Notice that a game with parameters in R4 is the limit of games with parameters in R2 as the spillovers become symmetric.

\section{Applications}

We now are in a position to show how our characterization of symmetric equilibria may be used to obtain closed-form expressions for equilibrium strategies in economic environments that include auctions, contests, and pricing games. In so doing, we also show that our results may be used to generalize existing models to allow for spillover effects, and to examine the implications of alternative behavioral and evolutionary 
assumptions on equilibrium. Throughout this section, we suppress ties, which we assume are broken with the flip of a fair coin.

\subsection{Auctions and contests with spillovers}

In addition to standard auctions and contests (such as first- and second-price auctions, the war of attrition, and the all-pay auction), our framework may be used to characterize symmetric equilibria in more exotic economic applications.

\subsubsection{Partnership dissolution (The self-auction)}

Two partners wish to dissolve a partnership each values at $v>0$. They simultaneously submit bids; the high bidder wins the asset and pays his bid to the other partner. Here, the payoffs are given by

$$
u_{i}\left(x_{i}, x_{j}\right)= \begin{cases}v-x_{i} & \text { if } x_{i}>x_{j} \\ x_{j} & \text { if } x_{i}<x_{j}\end{cases}
$$

and thus the game may be viewed as a $\Gamma$ with $V=v, \beta=-\theta=1, \alpha=\delta=0$, and $\eta=-2$. It follows from Proposition 1 that the only symmetric pure-strategy equilibrium is $x^{*}=v / 2$. Furthermore, Proposition 3 reveals that the self-auction does not have a non-degenerate symmetric mixed-strategy equilibrium.

\subsubsection{An innovation contest with spillovers}

One may also use our results to extend Dasgupta's (1986) all-pay auction formulation of an $R \& D$ race by allowing each firm's expenditure on $R \& D$ to induce an informational spillover that benefits the rival. In particular, suppose the winner receives a greater benefit per unit of expenditure from the loser's expenditure than the loser receives from the winner's expenditure. The corresponding payoffs are

$$
u_{i}\left(x_{i}, x_{j}\right)= \begin{cases}v-x_{i}-\delta x_{j} & \text { if } x_{i}>x_{j} \\ -x_{i}-\theta x_{j} & \text { if } x_{i}<x_{j}\end{cases}
$$

This game may be viewed as a $\Gamma$ in which $V=v>0, \alpha=\beta=1$, and $\delta<\theta<0$. Since $\alpha-\beta=0$ and $\eta>0$, Propositions 2 and 3 imply that the unique symmetric equilibrium is in non-degenerate mixed strategies (with parameters in R2) and is given by

$$
F^{*}(x)=\frac{1}{\theta-\delta} \ln \left(1+\frac{\theta-\delta}{v} x\right) \text { on }\left[0, \frac{v}{\theta-\delta}\left(\exp \left(\frac{\theta-\delta}{\alpha}\right)-1\right)\right] .
$$

\subsubsection{Territorial contests with injuries}

Next, consider a generalization of an all-pay auction formulation of a territorial contest in which the outcome of the battle depends on the intensity of effort put forth by the 
two players in the fight, where each player values the territory in dispute at $v>0$. Suppose the cost to a player per unit of intensity of effort is unity $(\alpha=\beta=1)$, and each unit of effort a player expends in the battle imposes a cost on its rival (through injury), so that $\delta, \theta>0$. If the cost to the loser per unit of intensity of effort of the winner is greater than the cost to the winner per unit of intensity of effort of the loser $(\theta>\delta>0)$, then $\alpha-\beta=0$ and $\eta=\theta-\delta>0$. In this case, Proposition 2 reveals that the symmetric equilibrium of this game is identical to that in the above innovation contest with spillovers.

If, on the other hand, the efforts of the winner and loser entail symmetric injury $(\theta=\delta>0)$, Proposition 2 implies that the symmetric equilibrium mixed-strategy (with parameters in R4) is given by

$$
F^{*}(x)=x / v \text { on }[0, v]
$$

which corresponds to the all-pay auction. However, expected payoffs are not zero (as they are in the standard all-pay auction). Notice that, as a result of spillovers, both of these variants differ from the classic war of attrition $(\beta=\theta=0$ and $\alpha=\delta=1)$, which lies in the region of R3 that entails an equilibrium distribution that is an exponential distribution.

\subsubsection{Litigation contests with fee-shifting}

Our framework may also be used to characterize symmetric equilibria for the complete information analogs of the Baye et al. (2005) model of litigation contests under incomplete information. In this application, players are litigants who compete by choosing (quality normalized) expenditures on legal services, with the player spending the higher amount winning the case valued at $v>0$. The fee-shifting rules examined by Baye et al. may be examined under complete information using the tools developed in Sect. 2.

For instance, under the Continental rule, the loser pays his own legal expenditure and, additionally, reimburses the winner a fraction $(1-\beta) \in(0,1)$ of the winner's legal expenditures. Thus, the Continental rule is a $\Gamma$ with $V=v, 0<\beta<\alpha=1$, and $\delta=0<\theta=(1-\beta)$. Since $\alpha>0, \beta>0, \alpha-\beta>0$, and $\eta=2(1-\beta)>0$, Proposition 2 implies that the corresponding equilibrium (with parameters in R1) is

$$
F^{*}(x)=\frac{1}{1-\beta}\left(1-\left(\frac{v}{v+2(1-\beta) x}\right)^{\frac{1}{2}}\right) \text { on }\left[0, \frac{v}{2(1-\beta)}\left(\frac{1}{\beta^{2}}-1\right)\right]
$$

and the equilibrium payoff is $E U^{*}=-v(1-\beta) /(2 \beta)<0$.

In contrast, under the Quayle rule, where the loser reimburses the winner up to the amount actually spent by the loser, $V=v \alpha=2, \beta=1, \delta=-1$ and $\theta=0$. Since $\alpha>0, \beta>0, \alpha-\beta>0$ and $\eta=2>0$, Proposition 2 implies that the 
corresponding equilibrium (with parameters in R1) is

$$
F^{*}(x)=2\left[1-\left(\frac{v}{v+2 x}\right)^{\frac{1}{2}}\right] \text { on }\left[0, \frac{3}{2} v\right]
$$

and the equilibrium payoff is $E U^{*}=0$. Furthermore, Proposition 1 reveals that symmetric pure-strategy equilibria do not arise in these litigation environments.

\subsection{Price competition}

We mentioned earlier that our framework readily includes standard models of price competition under complete information that take the form of first- and second-price auctions (which have a unique symmetric equilibrium in pure strategies) as well as variants that have symmetric mixed-strategy equilibria. We next show that our framework also subsumes several models of price competition in the industrial organization literature.

\subsubsection{Bertrand competition}

Our framework may be used to identify all symmetric equilibria in winner-take-all homogeneous product Bertrand games, including the unique symmetric pure-strategy equilibrium (marginal cost pricing) and the continuum of positive-profit mixed strategy equilibria identified by Baye and Morgan (1999). To see this, suppose two price-setting firms produce a homogeneous product at a constant marginal cost, $c>0$, and the firm offering the lowest price captures the entire market demand, which is given by $D(p)=1$ for $p \in[0, \infty)$. Thus, the profits of firm $i$ are given by

$$
\pi_{i}\left(p_{i}, p_{j}\right)= \begin{cases}0 & \text { if } p_{i}>p_{j} \\ p_{i}-c & \text { if } p_{i}<p_{j}\end{cases}
$$

Letting $x_{i} \equiv p_{i}$, this is a $\Gamma$ with $V=c, \alpha=-1, \beta=\theta=\delta=0$, and $\eta=-1$. By Proposition $1, x^{*} \equiv p^{*}=-V / \eta=c$ is the unique symmetric pure strategy Nash equilibrium (and each firm earns zero profits). By Proposition 2, there is also a continuum of symmetric positive profit equilibria in non-degenerate mixed strategies (with parameters in $\mathrm{R} 1$ ): For every $m \in(c, \infty)$,

$$
F^{*}(p)=1-\left(\frac{m-c}{p-c}\right) \text { on }[m, \infty)
$$

is a symmetric non-degenerate mixed-strategy equilibrium with corresponding equilibrium payoffs of $E U^{*}=m \in(c, \infty)$. 


\subsubsection{The Varian/Rosenthal model}

The price setting models of Varian (1980) and Rosenthal (1980) may be analyzed in our framework as follows. Here, two price-setting firms each service a fixed number, $L>0$, of loyal (or uninformed) consumers who have unit demand up to a choke price, $r>0$. Additionally, there are $S>0$ price sensitive "shoppers" (or informed consumers) who always purchase from the firm charging the lowest price-provided it does not exceed $r$. Each firm produces at zero cost to earn a payoff of

$$
\pi_{i}\left(p_{i}, p_{j}\right)= \begin{cases}(S+L) p_{i} & \text { if } p_{i}<p_{j} \\ L p_{i} & \text { if } p_{i}>p_{j}\end{cases}
$$

where, for simplicity we have suppressed the fact that a firm's profits are zero if it prices above $r$.

To see that this model is a special case of our framework, define $x_{i} \equiv r-p_{i} \geq 0$ so that the above payoffs are equivalent to those in a game in which

$$
u_{i}\left(x_{i}, x_{j}\right)= \begin{cases}(S+L) r-(S+L) x_{i} & \text { if } x_{i}>x_{j} \\ r L-L x_{i} & \text { if } x_{i}<x_{j}\end{cases}
$$

Thus, the Varian/Rosenthal models may be interpreted as a $\Gamma$ with $v=(S+L) r, \gamma=$ $-r L, V=r S>0, \beta=(S+L)>0, \delta=\theta=0, \alpha=L>0$, and $\eta=-S<0 .{ }^{10}$ Hence, by Proposition 2, the equilibrium (expressed in terms of the discount from the monopoly price, or $x \equiv r-p$ ) is associated with parameters in $\mathrm{R} 1$ and is given by

$$
F^{*}(x)=\frac{L}{S}\left(\frac{r}{r-x}-1\right) \text { on }\left[0, \frac{r S}{S+L}\right] .
$$

To write this expression in terms of the equilibrium distribution of prices, $G^{*}(p)$, use the fact that $x \equiv r-p$ and note that $G^{*}(p)=\operatorname{Pr}(P \leq p)=1-\operatorname{Pr}(x<r-p)=$ $1-F^{*}(r-p)$, so that

$$
G^{*}(p)=1-\frac{L}{S}\left(\frac{r-p}{p}\right) \text { on }\left[r \frac{L}{S+L}, r\right] .
$$

\subsubsection{Price matching guarantees}

One may also use our results to extend the Varian/Rosenthal models to allow for price matching policies, as in Png and Hirshleifer (1987) and Baye and Kovenock (1994). To see this, extend the Varian/Rosenthal models by assuming that the two firms not only list prices, but also promise to match a better price by the rival. Here, one interprets $S$ as informed consumers who are aware of the firms' prices, $L$ as uninformed consumers

\footnotetext{
10 Application of our Propositions also requires the strategy space in the transformed game to be $[0, \infty)$. Notice that $x_{i} \in[0, \infty)$ implies $p_{i} \in(-\infty, r]$. It is immediate that negative prices are strictly dominated. Hence, expanding the strategy space in the original game to include negative prices does not change the equilibrium set.
} 
who are unaware of the firms' prices, and one assumes that only a proportion $\mu<1 / 2$ of the informed customers are willing to bear the cost of visiting a firm charging the highest price to take it up on its offer to match a better price. Consequently, the firms' payoffs are

$$
\pi_{i}\left(p_{i}, p_{j}\right)=\left\{\begin{array}{ll}
(L+(1-\mu) S) p_{i} & \text { if } p_{i}<p_{j} \\
L p_{i}+\mu S p_{j} & \text { if } p_{i}>p_{j}
\end{array} .\right.
$$

As before, let $x_{i}=r-p_{i}$, so that the payoffs may be rewritten as

$$
u_{i}\left(x_{i}, x_{j}\right)=\left\{\begin{array}{ll}
r((1-\mu) S+L)-((1-\mu) S+L) x_{i} & \text { if } x_{i}>x_{j} \\
r(L+\mu S)-L x_{i}-\mu S x_{j} & \text { if } x_{i}<x_{j}
\end{array} .\right.
$$

Note that $V=(1-2 \mu) S r>0, \beta=(1-\mu) S+L>0, \alpha=L>0, \theta=S \mu>0$, and $\delta=0$. In this case, $\alpha-\beta=-(1-\mu) S<0$ and $\eta=-(1-2 \mu) S<0$, and the parameters lie in R1. Proposition 2 implies

$$
\begin{aligned}
F^{*}(x) & =\frac{L}{(1-\mu) S}\left(\left(\frac{r}{r-x}\right)^{\frac{(1-\mu)}{(1-2 \mu)}}-1\right) \text { on } \\
& {\left[0, r\left(1-\left(\frac{L}{((1-\mu) S+L)}\right)^{\frac{(1-2 \mu)}{(1-\mu)}}\right)\right] . }
\end{aligned}
$$

As before, one may easily re-write this distribution of discounts from the monopoly price purely in terms of the prices.

\subsection{Behavioral and evolutionary extensions}

The results in Sect. 2 may also be used to extend existing models to account for behavioral or evolutionary considerations that impact the payoffs in standard games. We discuss these applications next.

\subsubsection{Reference pricing}

One may use our results to analyze an extension of the Varian/Rosenthal models to account for reference pricing. To see this, suppose that in addition to shoppers and loyal consumers, there also exists a measure of "relative bargain seekers". As above, all consumer segments have a common choke price, $r>0$. Relative bargain seekers, like shoppers, always purchase from the firm offering the lowest price. But unlike shoppers, the demand of relative bargain seekers depends on how low the best price is in comparison to the next best price: The lower the "best" price relative to that of the higher price, the greater their demand for the low-priced good. To capture this behavior, assume that when firm $i$ charges the lowest price, its demand from relative bargain seekers is $D_{i} \equiv \lambda p_{j} / p_{i}$ while firm $j$ 's demand from these consumers is zero. 
When $\lambda>0$, this captures the behavioral phenomenon where the demand by one segment of consumers depends, in part, on their frame of reference.

With this extension, the payoffs are

$$
\pi_{i}\left(p_{i}, p_{j}\right)=\left\{\begin{array}{ll}
\left(S+L+\lambda \frac{p_{j}}{p_{i}}\right) p_{i} & \text { if } p_{i}<p_{j} \\
L p_{i} & \text { if } p_{i}>p_{j}
\end{array} .\right.
$$

As before, let $x_{i} \equiv r-p_{i}$, so that these payoffs are equivalent to

$$
u_{i}\left(x_{i}, x_{j}\right)=\left\{\begin{array}{ll}
r(S+L+\lambda)-x_{i}(S+L)-\lambda x_{j} & \text { if } x_{i}>x_{j} \\
r L-L x_{i} & \text { if } x_{i}<x_{j}
\end{array} .\right.
$$

If $\lambda \leq L$, this extension may be viewed as a $\Gamma$ with $V=r(S+\lambda)>0, \alpha=L>$ $0, \beta=S+L>0, \theta=0, \delta=\lambda, \alpha-\beta=-S<0$ and $\eta=-(S+\lambda)<0 .{ }^{11}$ These parameters lie in R1, and Proposition 2 implies

$$
F^{*}(x)=\frac{L}{S}\left(\left(\frac{r}{r-x}\right)^{\frac{S}{S+\lambda}}-1\right) \text { on }\left[0, r\left(1-\left(\frac{L}{S+L}\right)^{\frac{(S+\lambda)}{S}}\right)\right]
$$

or in terms of price,

$$
G^{*}(p)=1-\frac{L}{S}\left(\left(\frac{r}{p}\right)^{\frac{S}{S+\lambda}}-1\right) \text { on }\left[r\left(\frac{L}{S+L}\right)^{\frac{(S+\lambda)}{S}}, r\right]
$$

This distribution converges to that in the Varian/Rosenthal model as $\lambda$ tends to zero.

Interestingly, when there are only loyal customers and relative bargain seekers $(S=0)$, then $\eta=-\lambda<0$ and $\alpha-\beta=0$. In this case the parameters lie in R2, and Proposition 2 implies

$$
F^{*}(x)=\frac{L}{\lambda} \ln \frac{r}{r-x} \text { on }\left[0,\left(1-\exp \left(-\frac{\lambda}{L}\right)\right) r\right] \text {. }
$$

\subsubsection{Effort inequality aversion in a job tournament}

Next, consider an environment where two workers compete in a job tournament but exhibit a specialized form of inequality aversion such that they receive disutility from inequality of effort. We model inequality aversion with a utility function similar to that in Fehr and Schmidt (1999), except that our focus is on effort inequality rather than income inequality.

\footnotetext{
$\overline{11}$ Notice that $x_{i} \in[0, \infty)$ implies $p_{i} \in(-\infty, r]$. If $\lambda \leq L$, it is immediate that negative prices are strictly dominated. Hence, expanding the strategy space in the original game to include negative prices does not change the equilibrium set.
} 
In particular, suppose that the worker exerting the greater effort $\left(x_{i}\right)$ receives a bonus valued at $\mu>0$ and that payoffs are

$$
u_{i}\left(x_{i}, x_{j}\right)= \begin{cases}\mu-x_{i}-b\left(x_{i}-x_{j}\right) & \text { if } x_{i}>x_{j} \\ -x_{i}-a\left(x_{j}-x_{i}\right) & \text { if } x_{i}<x_{j}\end{cases}
$$

where $0<a<1$ and $0<b$. This captures behavior where the winner experiences disutility for having "slaughtered" the loser, and the loser derived disutility from having been beaten badly. In this case, utilities may be written as

$$
u_{i}\left(x_{1}, x_{2}\right)= \begin{cases}\mu-(1+b) x_{i}+b x_{j} & \text { if } x_{i}>x_{j} \\ -(1-a) x_{i}-a x_{j} & \text { if } x_{i}<x_{j}\end{cases}
$$

This behavioral environment may thus be viewed as a $\Gamma$ in which $V=v=\mu>$ $0, \gamma=0, \alpha=1-a>0, \theta=a, \beta=1+b>0$ and $\delta=-b<0$. Note that $\alpha-\beta=-(a+b)<0$ and $\eta=0$.

Since $\alpha=1-a>0$, it is immediate from Proposition 1 that there does not exist a symmetric pure strategy equilibrium. However, Propositions 2 and 3 imply that a unique non-degenerate symmetric mixed strategy equilibrium (with parameters in R3) exists. The corresponding distribution of effort is

$$
F^{*}(x)=\frac{1-a}{a+b}\left(\exp \left(\frac{a+b}{\mu} x\right)-1\right) \quad \text { on }\left[0, \frac{\mu}{a+b} \ln \left(\frac{1+b}{1-a}\right)\right]
$$

and each player earns an expected equilibrium payoff of

$$
E U^{*}=\frac{a}{a+b}\left[1+\frac{1+b}{a+b} \ln \left(\frac{1-a}{1+b}\right)\right] \mu .
$$

It is interesting to note that if $a \in(0,1)$ but $b \in(-1,0)$ (so that the winner enjoys "slaughtering" the loser), the equilibrium strategies and payoffs have exactly the same form when $b \neq-a$. But when $b=-a$ (so that $\alpha-\beta=0$ and $\eta=0$ ), the parameters lie in R4 and the equilibrium distribution of effort is identical to that in the all-pay auction:

$$
F^{*}(x)=\frac{1-a}{\mu} x \text { on }\left[0, \frac{\mu}{1-a}\right] \text {. }
$$

However, unlike the all-pay auction,

$$
E U^{*}=-\frac{a}{2(1-a)} \mu .
$$




\subsubsection{Loss aversion in a job tournament}

Two workers compete in a job tournament and the worker exerting the greater effort $\left(x_{i}\right)$ receives a bonus valued at $\mu>0$. Thus, their income is

$$
y_{i}= \begin{cases}\mu-x_{i} & \text { if } x_{i}>x_{j} \\ -x_{i} & \text { if } x_{i}<x_{j}\end{cases}
$$

Suppose the workers' utility (over income) exhibits "loss aversion" in that $u_{i}=y_{i}$ if player $i$ wins, and $u_{i}=\lambda y_{i}$ if player $i$ loses, where $\lambda>1$. In this case, utility (as a function of effort) is

$$
u_{i}\left(x_{i}, x_{j}\right)= \begin{cases}\mu-x_{i} & \text { if } x_{i}>x_{j} \\ -\lambda x_{i} & \text { if } x_{i}<x_{j}\end{cases}
$$

and this scenario may be analyzed as a $\Gamma$ with $v=\mu, \gamma=0, V=\mu>0, \alpha=$ $\lambda>0, \beta=1>0, \theta=\delta=0$, and $\eta=\alpha-\beta=\lambda-1>0$. Hence, Propositions 2 and 3 imply that the unique non-degenerate symmetric mixed-strategy equilibrium (with parameters in R1) is given by

$$
F^{*}(x)=\frac{\lambda x}{\mu+(\lambda-1) x} \text { on }[0, \mu] .
$$

\subsubsection{Regret in auctions}

A growing literature has examined regret in auctions; see Engelbrecht-Wiggans (1989), Engelbrecht-Wiggans and Katok (2007), and Filiz-Ozbay and Ozbay (2007) and the references cited therein. To illustrate how our framework may be used to examine the implications of this behavioral assumption in complete information environments, consider a first-price auction where each player $i \in\{1,2\}$ values the item at $v$, but there is winner regret such that the payoffs are

$$
u_{i}\left(x_{1}, x_{2}\right)=\left\{\begin{array}{ll}
v-x_{i}-\mu\left(x_{i}-x_{j}\right) & \text { if } x_{i}>x_{j} \\
0 & \text { if } x_{i}<x_{j}
\end{array} .\right.
$$

Here, $x_{i}$ is player $i$ 's bid and $v>0$ is the value of the item; winner regret $(\mu>0)$ refers to the fact that the high bidder derives disutility from leaving money on the table (the difference between the winning and losing bid). The payoffs may be rewritten as

$$
u_{i}\left(x_{1}, x_{2}\right)=\left\{\begin{array}{ll}
v-(\mu+1) x_{i}+\mu x_{j} & \text { if } x_{i}>x_{j} \\
0 & \text { if } x_{i}<x_{j}
\end{array},\right.
$$

which is a rank-order contest, $\Gamma$, with a positive first-order spillover effect. In particular, $V=v, \alpha=\theta=0, \beta=(1+\mu)>0, \delta=-\mu$, and $\eta=-1$, so Proposition 1 implies that a symmetric pure strategy Nash equilibrium exists and is given by $x^{*}=v$. Furthermore, by Proposition 3, there are no symmetric mixed-strategy equilibria. 
In a first-price auction with loser regret, payoffs are

$$
u_{i}\left(x_{1}, x_{2}\right)= \begin{cases}v-x_{i} & \text { if } x_{i}>x_{j} \\ -\rho\left(v-x_{j}\right) & \text { if } x_{i}<x_{j}\end{cases}
$$

where $\rho>0$. Preferences with loser regret thus transform the standard auction into a rank-order contest with a positive second-order spillover effect, and one can use the results in Sect. 2 to conclude that the unique symmetric equilibrium (pure or mixed) is $x^{*}=v$.

The results of Sect. 2 may also be used to extend these behavioral models to include combined winner and loser regret in a first-price auction. In this case, both first and second order spillover effects arise, as the payoffs are

$$
u_{i}\left(x_{1}, x_{2}\right)=\left\{\begin{array}{ll}
v-(\mu+1) x_{i}+\mu x_{j} & \text { if } x_{i}>x_{j} \\
-\rho\left(v-x_{j}\right) & \text { if } x_{i}<x_{j}
\end{array} .\right.
$$

One can readily establish that the unique symmetric equilibrium is in pure strategies and is given by $x^{*}=v$.

Furthermore, our results may be utilized to examine the implications of combined winner-loser regret in other auction environments. For instance, in an all-pay auction with winner-loser regret, payoffs are

$$
u_{i}\left(x_{1}, x_{2}\right)=\left\{\begin{array}{ll}
v-(\mu+1) x_{i}+\mu x_{j} & \text { if } x_{i}>x_{j} \\
-v \rho-(\rho+1) x_{i}+\rho x_{j} & \text { if } x_{i}<x_{j}
\end{array} .\right.
$$

This may be viewed as a $\Gamma$ in which $V=(1+\rho) v, \alpha=(1+\rho)>0, \beta=$ $(1+\mu)>0, \theta=-\rho, \delta=-\mu$, and $\eta=0$. When $\rho \neq \mu$, Propositions 2 and 3 imply that the unique symmetric equilibrium (with parameters in R3) is

$$
F^{*}(x)=\left(\frac{1+\rho}{\rho-\mu}\right)\left(1-\exp \left(-\frac{\rho-\mu}{1+\rho} \frac{x}{v}\right)\right) \text { on }\left[0, \frac{1-\delta}{\delta-\mu} \ln \left(\frac{1+\rho}{1+\mu}\right)\right]
$$

and each player earns an expected payoff of

$$
E U^{*}=v \rho+\rho(1+\rho) v \frac{(1+\mu) \ln \frac{1+\mu}{1+\rho}+\rho-\mu}{(\rho-\mu)^{2}} .
$$

However, if $\rho=\mu$, so that $\alpha=\beta$, one obtains the standard all-pay auction form (with parameters in R4): $F^{*}(x)=x / v$. In this case, total expected effort is the same with symmetric winner-loser regret as in the standard all-pay auction, but $E U^{*}=-\rho v / 2$.

\subsubsection{ESS in the all-pay auction}

Finally, one may utilize our results to construct equilibrium strategies in certain evolutionary environments. To see this, consider a two-player all-pay auction and note 
that the (finite agent) ESS equilibrium of Schaffer (1988) requires that each player maximizes the difference in payoffs. Thus

$$
u_{i}\left(x_{1}, x_{2}\right)=\left\{\begin{array}{ll}
v-x_{i}-\left(-x_{j}\right) & \text { if } x_{i}>x_{j} \\
-x_{i}-\left(v-x_{j}\right) & \text { if } x_{i}<x_{j}
\end{array} .\right.
$$

Hence, this may be viewed as a $\Gamma$ where payoffs are

$$
u_{i}\left(x_{1}, x_{2}\right)= \begin{cases}v-x_{i}+x_{j} & \text { if } x_{i}>x_{j} \\ -v-x_{i}+x_{j} & \text { if } x_{i}<x_{j}\end{cases}
$$

and $V=2 v>0, \beta=\alpha=-\theta=-\delta=1$, and consequently, $\alpha-\beta=0$ and $\eta=0$. Notice that these parameters lie in R4. One may therefore apply the results in Sect. 2 to conclude that the unique symmetric Nash equilibrium to a game with these payoffs (which corresponds to the ESS equilibrium of a game with the original formulation of payoffs) is

$$
F^{*}(x)=\frac{x}{2 v} \text { on }[0,2 v]
$$

Among other things, this implies that there is overdissipation of rents in the ESS equilibrium. This is similar to the findings of Hehenkamp et al. (2004) for the case of a Tullock contest.

\section{Conclusion}

This paper has characterized symmetric equilibria (pure and mixed) in a parameterized class of two player complete information contests with rank-order spillovers. We derived explicit closed form solutions for the complete set of symmetric equilibrium strategies for this class of games, and showed that these strategies take on only a small number of functional forms that depend on the parameters in a systematic and easily verified way. We concluded by using this framework to formulate and solve several new contests. Not only are a plethora of existing models of auctions, contests, and price competition covered as special cases, but our results permit one to extend these models to allow for a broader array of preferences, spillover effects, and equilibrium concepts. The logarithmic equilibrium distribution that arises in the all-pay auction with asymmetric spillovers, for example, appears to be novel to the literature. We believe that Propositions 1 through 3 will provide positive spillovers for future applied work on auctions, contests, and pricing strategies, as well as behavioral economics and evolutionary game theory.

\section{Appendix}

This Appendix provides the proofs of Propositions 1 through 3. Recall the definitions of $W, L$, and $T$ are given in Eq. (1). 


\section{A1. Proof of Proposition 1}

The following lemma is useful in proving Proposition 1.

Lemma $1 x \in[0, \infty)$ is a symmetric pure strategy Nash equilibrium of $\Gamma$ if and only if the following two conditions hold:

$$
\begin{aligned}
& T(x, x) \geq W(y, x) \text { for all } y \geq x \\
& T(x, x) \geq L(y, x) \text { for all } 0 \leq y \leq x .
\end{aligned}
$$

Proof Recall that $x_{1}=x_{2}=x$ is a symmetric pure strategy Nash equilibrium if and only if $u_{i}(x, x) \geq u_{i}(y, x)$ for all $y \in[0, \infty)$. Note that $u_{i}(y, x)=L(y, x)$ for $y<x$ and $u_{i}(y, x)=W(y, x)$ for $y>x$. Additionally, since

$$
u_{i}(x, x)=T(x, x)=\frac{1}{2} W(x, x)+\frac{1}{2} L(x, x)
$$

the conditions in (8) imply

$$
u_{i}(x, x)=T(x, x)=W(x, x)=L(x, x) .
$$

$(\Longleftarrow)$ By hypothesis, $x \in[0, \infty)$ satisfies

$$
\begin{aligned}
& T(x, x) \geq W(y, x) \quad \text { for all } y \geq x \\
& T(x, x) \geq L(y, x) \quad \text { for all } y \leq x .
\end{aligned}
$$

Hence, if player $i$ plays the pure strategy $x_{i}=x$ when player $j$ plays $x_{j}=x$, she earns a payoff of $U^{*}=T(x, x)=W(x, x)=L(x, x)$. The conditions in (8) imply that player $i$ cannot gain by deviating from $x$, given that $x_{j}=x$.

$(\Longrightarrow)$ If $(x, x)$ is a symmetric pure strategy Nash equilibrium, player $i$ earns a payoff of $T(x, x)$ in this equilibrium. By way of contradiction, suppose there exists a $y \in[0, \infty)$ such that $y>x$ with $T(x, x)<W(y, x)$. Then player $i$ could increase his payoff to $W(y, x)>T(x, x)$ by deviating from $x_{i}=x$ to $x_{i}=y$, a contradiction. Similarly, if there existed a $y \in[0, \infty)$ such that $y<x$ with $T(x, x)<L(y, x)$, player $i$ could increase his payoff to $L(y, x)>T(x, x)$ by deviating from $x_{i}=x$ to $x_{i}=y$, a contradiction.

We conclude that the conditions in (8) are necessary and sufficient for the existence of a symmetric pure strategy Nash equilibrium. Note that the proof of Lemma 1 does not rely on the linear structure for $W$ and $L$ in Eq. (1), and hence applies to more general formulations for payoffs.

We are now in a position to prove Proposition 1. We do so by exploiting the linear structure in Eq. (1) and applying Lemma 1.

Proof of Proposition $1(\Longrightarrow)$ By way of contradiction, suppose $x \in[0, \infty)$ is a symmetric pure strategy Nash equilibrium so that player $i$ earns his equilibrium payoff of 
$U^{*}=T(x, x)=W(x, x)=L(x, x)$ at $(x, x)$. If condition $(i)$ in Proposition $1 \mathrm{did}$ not hold, then player $i$ could deviate to earn $W(x+\varepsilon, x)>U^{*}=W(x, x)$, since $\beta<0$ implies $W\left(x_{i}, x\right)$ is increasing in $x_{i}$, a contradiction. If condition (iii) did not hold, then $V+\eta x=W(x, x)-L(x, x)>0$, which contradicts the conditions in (8). Finally, since $V>0$, condition (iii) implies $x>0$. Thus, if condition (ii) did not hold, then $x>0$ and $\alpha>0$, in which case player $i$ could deviate to earn a payoff of $L(x-\varepsilon, x)>L(x, x)=W(x, x)=U^{*}$, since $\alpha>0$ implies $L\left(x_{i}, x\right)$ is decreasing in $x_{i}$, a contradiction.

$(\Longleftarrow)$ Suppose conditions $(i)$ through $(i i i)$ hold. It is immediate that condition (iii) implies that $x^{*}=-V / \eta$ is well-defined and $V+\eta x^{*}=W\left(x^{*}, x^{*}\right)-L\left(x^{*}, x^{*}\right)=0$. Hence, $W\left(x^{*}, x^{*}\right)=L\left(x^{*}, x^{*}\right)=T\left(x^{*}, x^{*}\right)$. Next, note that there is no incentive to deviate from $x^{*}$, since (i) implies $T\left(x^{*}, x^{*}\right) \geq W\left(y, x^{*}\right)$ for all $y \geq x^{*}$, and (ii) implies $T\left(x^{*}, x^{*}\right) \geq L\left(y, x^{*}\right)$ for all $y \leq x^{*}$. By Lemma 1 , this implies that $x^{*}$ is a symmetric pure strategy Nash equilibrium. Uniqueness follows from Lemma 1 and the fact that $x^{*}=-V / \eta$ is the unique solution to $W(x, x)-L(x, x)=0$.

\section{A2. Proof of Proposition 2}

Our second proposition is proved through a sequence of lemmas. We first demonstrate that if an atom exists at some point $(x, x)$ in a non-degenerate symmetric mixedstrategy equilibrium of $\Gamma$, then $(x, x)$ constitutes a symmetric pure strategy equilibrium as well. We then apply Proposition 1 to show that this can occur only over a restricted range of the parameter space and that any such atom is unique. Consequently, if the symmetric equilibrium is in non-degenerate mixed strategies, there must exist an absolutely continuous part of the mixed-strategy, and furthermore, it must satisfy the differential equation in Eq. (3). Given the linearity of differential equation (3), it readily follows that its solution over the interval $(m, u)$ is unique (as it satisfies a Lipschitz condition). Lemma 4 provides an endpoint restriction on the lower bound of the distribution. We then examine (by exhaustion) a partition of the parameter space to show (i) when the differential equation can be solved in a manner consistent with the corresponding restrictions on mass points and endpoints, (ii) which solutions indeed define equilibria in the sense that there is no incentive for a player to unilaterally deviate from his strategy, and (iii) whether the differential equation, mass point and endpoint restrictions are inconsistent, thereby implying nonexistence of a nondegenerate symmetric mixed-strategy. We also derive the corresponding equilibrium payoffs.

Lemma 2 If there is an atom at some point $x \in[0, \infty)$ in a non-degenerate symmetric mixed-strategy equilibrium of $\Gamma$, then $(x, x)$ is also a symmetric pure strategy equilibrium of $\Gamma$. Furthermore, there is no atom at $x=0$.

Proof If there is an atom of size $q_{x} \in(0,1)$ at some point $x$, it must be the case that $q_{x}[W(x+\varepsilon, x)-T(x, x)] \leq 0$ (there is no incentive to raise the bid above $\left.x\right)$ and, if in addition $x>0, q_{x}[L(x-\varepsilon, x)-T(x, x)] \leq 0$ for small $\varepsilon>0$ (there is no incentive to lower the bid below $x$ ). Furthermore, there can be no atom at $x=0$, since $q_{0}[W(0+\varepsilon, 0)-T(0,0)] \leq 0$ implies $W(0,0)-T(0,0) \leq 0$ by the continuity 
of $W$, contradicting $W(0,0)-T(0,0)=V / 2>0$. For $x>0$, since $q_{x}>0$ by hypothesis, $[W(x+\varepsilon, x)-T(x, x)] \leq 0$ and $[L(x-\varepsilon, x)-T(x, x)] \leq 0$. This implies $T(x, x)=W(x, x)=L(x, x)$, and furthermore, given the linearity of $W$ and $L$,

$$
\begin{aligned}
& T(x, x) \geq W(y, x) \quad \text { for all } y \geq x \\
& T(x, x) \geq L(y, x) \text { for all } y \leq x .
\end{aligned}
$$

These are exactly the conditions (8) for a pure strategy solution from Theorem 1 and hence $(x, x)$ must also be a pure strategy equilibrium point.

Lemma 3 Suppose a symmetric equilibrium strategy of $\Gamma$ has an atom of size $q_{x} \in$ $(0,1]$ at $x$. Then $\beta \geq 0, \alpha \leq 0$ and $\eta<0$. Furthermore the atom is unique and located at $x=-V / \eta>0$.

Proof Follows immediately from Lemma 2 and Proposition 1.

Importantly, Lemma 3 implies that if a non-degenerate symmetric mixed-strategy equilibrium exists, any atom (if one exists) associated with the strategy is necessarily unique (and given by $x=-V / \eta$ ). Consequently, the remaining absolutely continuous part is characterized by differential equation (3). We will use this fact to establish when non-degenerate symmetric mixed-strategy equilibria exist, their functional forms and the corresponding equilibrium payoffs. We also identify parameter configurations for which the set of non-degenerate symmetric mixed-strategy equilibria is empty. We also use this lemma to establish:

Lemma 4 Suppose $\alpha>0$. Then in any non-degenerate symmetric mixed-strategy equilibrium of $\Gamma$, the lower bound of the support is $m=0$.

Proof The proof proceeds by way of contradiction. Suppose the lower bound of the support of the equilibrium mixed-strategy is $m>0$ and let $q_{m}$ be the size of an atom (possibly zero) at $m$. Then a player who bids $m$ earns his equilibrium payoff of

$$
\begin{aligned}
U^{*} & =q_{m} T(m, m)+\left(1-q_{m}\right)\left(-\gamma-\alpha m-\theta E_{F}[x \mid x>m]\right) \\
& =\frac{q_{m}}{2} V-\gamma+\frac{q_{m}}{2} \alpha m-\alpha m-\frac{q_{m}}{2}(\theta+\beta+\delta) m-\left(1-q_{m}\right) \theta E_{F}[x \mid x>m] .
\end{aligned}
$$

Deviating by bidding zero yields a payoff of

$$
U^{* *}=-\gamma-\theta q_{m} m-\theta\left(1-q_{m}\right) E_{F}[x \mid x>m] .
$$

The difference in payoffs is thus

$$
U^{* *}-U^{*}=\frac{q_{m}}{2}\{-V+(-\theta-\alpha+\beta+\delta) m\}+\alpha m=\alpha m>0
$$

since $q_{m}>0$ implies $-V=\eta m$ by Lemma 3. Therefore it pays to deviate by bidding zero, a contradiction. 
We are now in a position to consider, case by case, the parameter configurations identified in Proposition 2. We do this through a sequence of lemmas that are collected according to the four parameter regions (R1 through R4) defining the different forms for the equilibrium mixed strategies in Eq. (5), and which establish existence, uniqueness, or nonexistence of equilibrium for parameter configurations within each case. We begin with

Case $1: \alpha-\beta \neq 0 ; \eta \neq 0$

Lemma 5 Suppose $\beta=0, \alpha>0$ and $\eta \neq 0$. Then there exists a symmetric equilibrium if and only if either $\theta=0$ or $\eta<\alpha$. Furthermore, this equilibrium is unique and in non-degenerate mixed strategies as characterized in Proposition 2.

Proof Since $\alpha>0$, Proposition 1 implies any symmetric equilibrium must be in non-degenerate mixed-strategies, and by Lemma 3, there are no atoms. By Lemma 4, $m=0$. Hence, if a symmetric equilibrium exists, it necessarily has the form in Eq. (4) with $C=0$ :

$$
F(x)=1-\left(\frac{V}{V+\eta x}\right)^{\alpha / \eta}
$$

This is a well-defined distribution function; when $\eta>0$, the upper bound of its support is $u=\infty$; when $\eta<0$, it is $u=-V / \eta<\infty$. Since $\beta=0$, a player cannot gain by choosing an action $w>u$. Thus, for an equilibrium to exist, it is sufficient to show that $E U^{*}<\infty$ and a player's expected payoff against a rival who uses $F$ is constant for any action in the support of $F$.

The expected payoff when a player chooses $x_{i}=w$ against such a strategy is

$$
E U(w)=\int_{0}^{w}(v-\delta x) \mathrm{d} F(x)+\int_{w}^{u}(-\gamma-\alpha w-\theta x) \mathrm{d} F(x)
$$

or, since this also holds at $w=0$,

$$
\begin{aligned}
E U(0) & =\int_{0}^{u}(-\gamma-\theta x) \mathrm{d} F(x) \\
& =-\gamma-\int_{0}^{u} \theta x \mathrm{~d} F(x) .
\end{aligned}
$$

Evidently, when $\theta=0, E U(0)=-\gamma$, so $E U(w)=-\gamma$ for all $w \in \operatorname{Support}(F)$, and $F$ is the unique symmetric equilibrium. Thus, suppose $\theta \neq 0$.

Consider first the case where $\eta>0$. In this case $u=\infty$ and the distribution in (9) has a Pareto-type "fat tail" and $\int_{0}^{u} x \mathrm{~d} F(x)$ is unbounded when $\alpha / \eta-1 \leq 0$ (or equivalently, $\delta \leq \theta \neq 0$ ). Thus, when $\beta=0, \theta \neq 0$ and $0<\alpha \leq \eta$, we conclude that $E U(0)$ is unbounded and hence there does not exist a symmetric equilibrium. 
But if $\alpha / \eta-1>0$ (or equivalently, $\delta>\theta \neq 0$ ), the relevant integral is finite and the expected payoff is

$$
E U^{*}=-\gamma-\theta \int_{0}^{\infty} x \alpha\left(\frac{V}{V+\eta x}\right)^{\alpha / \eta} \frac{1}{V+\eta x} \mathrm{~d} x=\frac{\theta v+\delta \gamma}{\theta-\delta}
$$

Since for all $w \in[0, \infty), E U(w)=E U^{*}$, in this case it follows that $F$ is the unique symmetric equilibrium.

Finally, if $\eta<0$, then $u=-V / \eta$ and simple integration reveals

$$
E U(0)=(\theta v+\delta \gamma) /(\theta-\delta)=E U(w),
$$

for all $w \in[0, u]$, and hence $F$ is the unique symmetric equilibrium.

Lemma 6 Suppose $\beta=0, \alpha<0$ and $\eta>0$. Then there does not exist a symmetric mixed-strategy equilibrium.

Proof Since $\alpha<0$ and $\eta>0$, Lemma 3 implies that $F$ contains no atoms. Hence, $C=0$ in Eq. (4), and thus

$$
F(w)=1-\left(\frac{V+\eta w}{V+\eta m}\right)^{-\alpha / \eta}
$$

But note that, since $-\alpha / \eta>0$ and $\eta>0$,

$$
\left(\frac{V+\eta w}{V+\eta m}\right)^{-\alpha / \eta}>1
$$

for all $w>m$, which implies $F(w) \leq 0$, a contradiction.

Lemma 7 Suppose $\beta=0, \alpha<0$ and either $\alpha<\eta<0$ or $\eta<\theta=0$. Then there exists a continuum of non-degenerate symmetric mixed-strategy equilibria, all of which are identified in Proposition 2. Furthermore, if $\beta=0, \alpha<0, \eta \leq \alpha$ and $\theta \neq 0$, there does not exist a non-degenerate symmetric mixed-strategy equilibrium.

Proof By Proposition 1, a unique symmetric pure-strategy equilibrium exists at $x^{*}=$ $-V / \eta$. By Lemma 3, in any non-degenerate symmetric mixed-strategy equilibrium, there is at most a single mass point, and this atom is located at $-V / \eta$. Let $q \in[0,1)$ denote the size of any such mass point. By way of contradiction, suppose that the lower bound of the absolutely continuous part of $F$ is $m>-V / \eta$ (that is, $F$ contains 
a gap). Then the expected payoff to a player that bids $-V / \eta$ against $F$ is

$$
\begin{aligned}
E U\left(\frac{V}{-\eta}\right)= & q T\left(\frac{V}{-\eta}, \frac{V}{-\eta}\right)+(1-q)\left(-\gamma-\alpha\left(\frac{V}{-\eta}\right)-\frac{1}{1-q} \int_{m}^{\infty} \theta x \mathrm{~d} F(x)\right) \\
= & \frac{q}{2}\left[v-\gamma+(\delta+\alpha+\theta) \frac{V}{\eta}\right] \\
& +(1-q)\left(-\gamma+\alpha \frac{V}{\eta}-\frac{1}{1-q} \int_{m}^{\infty} \theta x \mathrm{~d} F(x)\right) .
\end{aligned}
$$

A player that bids $m>-V / \eta$ against $F$ earns an expected payoff of

$$
E U(m)=q\left(v+\delta \frac{V}{\eta}\right)+(1-q)\left(-\gamma-\alpha m-\frac{1}{1-q} \int_{m}^{\infty} \theta x \mathrm{~d} F(x)\right) .
$$

Recall that $V=v+\gamma$ and that, under the conditions stated, $\eta=\alpha+\theta-\delta$. Provided $\int_{m}^{\infty} x \mathrm{~d} F(x)$ exists or $\theta=0$, straightforward algebra reveals

$$
E U(m)-E U\left(\frac{V}{-\eta}\right)=-\alpha(1-q)\left(m-\frac{V}{-\eta}\right)>0,
$$

which is a contradiction: there can be no atom below the lower bound of the absolutely continuous part of a symmetric mixed-strategy equilibrium.

We next show that under the conditions stated, $m>-V / \eta$ (which implies there are no mass points) and that there exists a continuum of symmetric equilibria of the form in Proposition 2. To see this, note that for $\alpha<0$ and $\eta<0$, Eq. (3) requires that $m>-V / \eta$ in order for $F(w)$ to be a well-defined (and non-degenerate) distribution function on an open interval above $m$. It follows that, when $\beta=0, \alpha<0$ and $\eta<0$, the only candidate for a non-degenerate symmetric mixed-strategy equilibrium is

$$
F(w)=1-\left(\frac{V+\eta m}{V+\eta w}\right)^{\alpha / \eta}
$$

on $[m, \infty)$, where $m \in(-V / \eta, \infty)$ is arbitrary. The expected payoff when a player chooses $x_{i}=w$ against $F$ is

$$
E U(w)=\int_{m}^{w}(v-\delta x) \mathrm{d} F(x)+\int_{w}^{\infty}(-\gamma-\alpha w-\theta x) \mathrm{d} F(x)
$$

If $\alpha / \eta \leq 1, \int_{m}^{\infty} x \mathrm{~d} F(x)$ is unbounded due to the fat (Pareto-type) tail of the distribution. In this case, when $\theta \neq 0, E U(w)$ is unbounded and hence a non-degenerate symmetric mixed-strategy equilibrium does not exist. But if $\theta=0$, the expected 
payoff to a player that bids $w=m$ is $E U(m)=-\gamma-\alpha m$, and hence for all $w \in[m, \infty), E U(w)=E U(m)$. Since $\alpha<0, E U(w)<E U(m)<\infty$ for $w<m$, and hence it does not pay to deviate by choosing an action below $m$.

When $\alpha / \eta>1$ (which implies $\theta>\delta$ ) it follows that for all $w \in[m, \infty$ ),

$$
\begin{aligned}
E U(w) & =E U(m)=-\gamma-\alpha m-\theta \int_{m}^{\infty} x \alpha\left(\frac{V+\eta m}{V+\eta x}\right)^{\alpha / \eta} \frac{1}{V+\eta x} \mathrm{~d} x \\
& =\frac{\theta v+\delta \gamma}{\theta-\delta}+\frac{\alpha \delta m}{\theta-\delta} .
\end{aligned}
$$

Again, since $\alpha<0, E U(w)<E U(m)<\infty$ for $w<m$, it does not pay to deviate by choosing an action below $m$.

Lemma 8 Suppose $\beta<0, \alpha \neq \beta, \alpha \neq 0$, and $\eta \neq 0$. Then there does not exist $a$ symmetric equilibrium.

Proof Since $\beta<0$, Proposition 1 implies there does not exist a symmetric pure strategy equilibrium, and by Lemma 3 , there are no mass points in a non-degenerate symmetric mixed-strategy equilibrium. Since $F(m)=0, C=0$ in Eq. (4); hence, if a symmetric mixed-strategy equilibrium exists, it must be of the form

$$
F(w)=\frac{\alpha}{\alpha-\beta}\left(1-\left(\frac{V+\eta m}{V+\eta w}\right)^{\frac{\alpha-\beta}{\eta}}\right) .
$$

We claim the distribution is unbounded. To see this, suppose to the contrary that $u<\infty$. Since $F$ has no atoms, a player that bids $u$ is certain to win and earn an equilibrium payoff of $E U(u)=v-\beta u-\delta E_{F}[x]$. But, since $\beta<0$, a player who deviates by bidding $u^{\prime}>u$ earns an expected payoff of $E U\left(u^{\prime}\right)=v-\beta u^{\prime}-\delta E_{F}[x]>E U(u)$, a contradiction.

If $(\alpha-\beta) / \eta>0$, Eq. (12) implies

$$
\lim _{w \rightarrow \infty} F(w)=\frac{\alpha}{\alpha-\beta} \neq 1
$$

If $(\alpha-\beta) / \eta<0$, then

$$
\lim _{w \rightarrow \infty} F(w)= \pm \infty
$$

Hence, regardless of the sign of $(\alpha-\beta) / \eta, F$ is not a well-defined distribution function, a contradiction. Thus there does not exist a symmetric mixed-strategy equilibrium in this case.

Lemma 9 Suppose $\beta>0, \alpha<0, \eta \neq 0$. Then there does not exist a non-degenerate symmetric mixed-strategy equilibrium. 
Proof If $\eta>0$, then there are no mass points by Lemma 3. A symmetric equilibrium, if one exists, must therefore satisfy Eq. (4) with $C=0$ :

$$
F(w)=\frac{\alpha}{\alpha-\beta}\left[1-\left(\frac{V+\eta m}{V+\eta w}\right)^{\frac{\alpha-\beta}{\eta}}\right] \text { for } w \in(m, u) .
$$

Since $(\alpha-\beta) / \eta<0$ and $\eta w>\eta m \geq 0$ for $w>m$, the term in square brackets is negative. This and the fact that $\alpha /(\alpha-\beta)>0$ implies $F(w)<0$, which contradicts the assumption that $F$ is a well-defined distribution function.

If $\eta<0$, Lemma 3 implies that an equilibrium mixed-strategy may have a mass point at $w=-V / \eta$ (hence $C \geq 0$ ). Hence, Eq. (4) implies

$$
F(w)=\frac{\alpha}{\alpha-\beta}+\left(C-\frac{\alpha}{\alpha-\beta}\right)\left[\frac{V+\eta m}{V+\eta w}\right]^{\frac{\alpha-\beta}{\eta}} \quad \text { for } w \in(m, u) .
$$

If the distribution is unbounded, $\lim _{w \rightarrow \infty} F(w)=\alpha /(\alpha-\beta)<1$, a contradiction. Thus, suppose $u<\infty$.

Suppose first that the equilibrium distribution contains no mass point. Then the differential equation in Eq. (3) holds at $u$, and since $F(u)=1$, we have

$$
[V+\eta u] f(u)-\alpha+(\alpha-\beta)=0 \text {. }
$$

Now, $f(u) \geq 0, \alpha<0, \beta>0$ and $\eta<0$ implies $u<-V / \eta$. Hence, the derivative of Eq. (13) is

$$
F^{\prime}(w)=\alpha(V+\eta m)^{\frac{\alpha-\beta}{\eta}}(V+\eta w)^{-\frac{\alpha-\beta+\eta}{\eta}}<0
$$

since $V+\eta w>0$ for all $w \in[m, u]$, a contradiction.

Finally, suppose there is a mass point. We first show the mass point must be located at or below $m$. By way of contradiction, suppose there is a mass point at $-V / \eta>m$. In this case, the differential equation (3) holds at $m$, and $F(m)=0$. Hence,

$$
[V+\eta m] f(m)-\alpha=0 .
$$

Since $f(m) \geq 0, \alpha<0$, and $0 \leq m<-V / \eta$, this is a contradiction.

Since the mass point must be at $-V / \eta$ and $m \geq-V / \eta$, the derivative of Eq. (4) is (for $w>m$ )

$$
F^{\prime}(w)=\left(\frac{\alpha}{\alpha-\beta}-C\right)(\alpha-\beta)\left(\frac{V+\eta m}{V+\eta w}\right)^{\frac{\alpha-\beta}{\eta}} \frac{1}{V+\eta w} .
$$

Since $F^{\prime}(w)>0$ for some $w>m$,

$$
\operatorname{sgn}\left(F^{\prime}(w)\right)=\operatorname{sgn}\left(\frac{\alpha}{\alpha-\beta}-C\right)>0
$$


in order for $F$ to be a well-defined distribution. Since the differential equation holds at $w=u$, setting $F(u)=1$ implies

$$
\frac{\beta}{\alpha}=\left[1-\frac{\alpha-\beta}{\alpha} C\right]\left(\frac{V+\eta m}{V+\eta u}\right)^{\frac{\alpha-\beta}{\eta}} .
$$

The LHS is strictly negative by assumption, while the RHS is strictly positive by Eq. (14) and the fact that $m, u>-V / \eta-$ a contradiction.

Hence, there does not exist a non-degenerate mixed-strategy equilibrium.

Lemma 10 Suppose $\beta>0, \alpha>0, \alpha \neq \beta$, and $\eta \neq 0$. Then there exists a unique symmetric equilibrium and it is in non-degenerate mixed-strategies as identified in Proposition 2.

Proof Since $\alpha>0$, Lemma 3 implies there are no mass points, and Lemma 4 implies $m=0$. Hence Eq. (4) implies

$$
F(w)=\frac{\alpha}{\alpha-\beta}\left[1-\left(\frac{V}{V+\eta w}\right)^{\frac{\alpha-\beta}{\eta}}\right] .
$$

It is straightforward to show that, for all $\beta>0, \alpha>0, \alpha \neq \beta$, and $\eta \neq 0$, this a well-defined distribution function on $\left[0, u^{*}\right]$, where

$$
u^{*}=\frac{V}{\eta}\left(\left(\frac{\alpha}{\beta}\right)^{\frac{\eta}{\alpha-\beta}}-1\right)>0 .
$$

Suppose first that $\theta \neq \delta$ (or equivalently, $\eta \neq \alpha-\beta$ ). The expected payoff to a player that bids $w=0$ against a rival that employs $F$ is

$$
\begin{aligned}
E U^{*} & =E U(w=0)=-\gamma-\theta \int_{0}^{u^{*}} x \mathrm{~d} F(x) \\
& =\frac{\theta v+\delta \gamma}{\theta-\delta}+\beta \frac{\theta}{\eta(\theta-\delta)}\left[1-\left(\frac{\alpha}{\beta}\right)^{\frac{\eta}{\alpha-\beta}}\right] V .
\end{aligned}
$$

Hence, $E U(w)=E U^{*}$ for all $w \in\left[0, u^{*}\right]$, and it does not pay to deviate to a $w>u^{*}$ since $\beta>0$.

When $\theta=\delta$ (or equivalently, $\eta=\alpha-\beta$ ), the expected payoff to a player that bids $w=0$ against a rival that employs $F$ is

$$
\begin{aligned}
E U(w=0) & =-\gamma-\theta \int_{0}^{u^{*}} x \mathrm{~d} F(x) \\
& =-\gamma+\frac{\theta V}{\eta}-\frac{\theta \alpha V}{\eta^{2}} \ln \frac{\alpha}{\beta}
\end{aligned}
$$


As above, since $\beta>0$, a player cannot gain by deviating to a $w>u^{*}$. We conclude that $F$ is the unique symmetric equilibrium in this case.

Lemma 11 Suppose $\alpha-\beta \neq 0, \alpha=0$ and $\eta \neq 0$. Then there does not exist a symmetric equilibrium in non-degenerate mixed strategies.

Proof Under the conditions stated, the solution to differential equation (3) is

$$
F(w)=K\left[\frac{V+\eta w}{V+\eta m}\right]^{\frac{\beta}{\eta}}
$$

for some $K \neq 0$. By hypothesis, $\beta \neq 0$. Suppose first that $\beta<0$. Then there is no mass point by Lemma 3, and hence $F(u)=1$ implies

$$
u=\frac{K^{-\frac{1}{\beta} \eta} V+K^{-\frac{1}{\beta} \eta} \eta m-V}{\eta} .
$$

But since $\beta<0$, this is a contradiction, since a player could improve his payoff by bidding above $u$.

Suppose next that $\beta>0$. If $\eta>0$ then once again there is no mass point by Lemma 3. Hence, $F(w)=0$ implies $w=-V / \eta$. But since $-V / \eta<0$, this is a contradiction.

Finally, suppose $\beta>0$ and $\eta<0$. Then

$$
\begin{aligned}
f(w) & =K(V+\eta w)^{\frac{\beta-\eta}{\eta}}(V+\eta m)^{-\frac{\beta}{\eta}} \beta \\
& =\beta \frac{F(w)}{V+\eta w}
\end{aligned}
$$

and hence $w<-V / \eta$. By Lemma 3, any mass point must be above the upper bound of the absolutely continuous part of $F$. Setting $F(w)=0$ in Eq. (16) implies the lower bound of the distribution must be $-V / \eta$. But this is a contradiction, since by Lemma 3 , the mass point must be located at this point.

Case $2: \alpha=\beta ; \eta \neq 0$

Lemma 12 Suppose $\alpha=\beta>0$ and $\eta \neq 0$. Then there exists a unique symmetric equilibrium and it is in non-degenerate mixed strategies as identified in Proposition 2.

Proof Note first that the conditions of the Lemma imply $\theta \neq \delta$. Since $\alpha>0$, Lemma 3 implies there are no mass points. Moreover, by Lemma 4, $\alpha>0$ implies $m=0$. Hence the differential equation in (3) implies

$$
f(w)=\frac{\alpha}{V+\eta w}
$$

which together with $F(m)=0$, implies that the unique $F$ is

$$
F(x)=\int_{0}^{x} \frac{\alpha}{V+\eta w} \mathrm{~d} w=\frac{\alpha}{\theta-\delta} \ln \left(\frac{V+(\theta-\delta) x}{V}\right)
$$


on $\left[0, \frac{V}{\theta-\delta}\left(\exp \left(\frac{\theta-\delta}{\alpha}\right)-1\right)\right]$, where we have used the fact $\eta=\theta-\delta$ under the conditions stated. The expected payoff of a player that bids $w=0$ is

$$
\begin{aligned}
E U(0) & =\int_{0}^{u}(-\gamma-\theta x) \frac{\alpha}{V+\eta x} \mathrm{~d} x \\
& =\frac{\theta v+\delta \gamma}{\theta-\delta}+\frac{\alpha \theta}{(\theta-\delta)^{2}}\left(1-\mathrm{e}^{\frac{\theta-\delta}{\alpha}}\right) V
\end{aligned}
$$

and hence $E U(w)=E U(0)$ for all $w \in\left[0, \frac{V}{\theta-\delta}\left(\exp \left(\frac{\theta-\delta}{\alpha}\right)-1\right)\right]$. Since $\beta>0$, a player cannot gain by bidding above the upper bound of the support.

Lemma 13 Suppose $\alpha=\beta<0$ and $\eta \neq 0$. Then there does not exist a symmetric mixed-strategy equilibrium.

Proof Lemma 3 implies there are no mass points, and hence Eq. (17) implies that, if an equilibrium exists, it must have the form

$$
\begin{aligned}
F(x) & =\int_{m}^{x} \frac{\alpha}{V+\eta w} \mathrm{~d} w \\
& =\frac{\alpha}{\theta-\delta} \ln \left(\frac{V+(\theta-\delta) x}{V+(\theta-\delta) m}\right),
\end{aligned}
$$

where we have used the fact that $\eta=\theta-\delta$ under the conditions stated. Since $F(u)=1$ implies $u<\infty$, the support of $F$ is bounded. But then $F$ cannot be part of a Nash equilibrium since a player can increase his expected payoff by bidding above $u$, as $\beta<0$.

Lemma 14 Suppose $\alpha=\beta=0$ and $\eta \neq 0$. Then there does not exist a symmetric mixed-strategy equilibrium.

Proof Under the conditions stated, differential equation (3) implies

$$
(V+\eta w) f(w)=0,
$$

which contradicts the hypothesis that there is a non-degenerate mixed-strategy.

Case 3: $\alpha-\beta \neq 0 ; \eta=0$

Lemma 15 Suppose $\eta=0, \alpha-\beta \neq 0$, and $\alpha<0$. Then there does not exist a symmetric equilibrium.

Proof First, note that since $\eta=0$, there can be no mass point by Lemma 3. Under the conditions stated, differential equation (3) implies

$$
V f(w)-\alpha+(\alpha-\beta) F(w)=0
$$


and hence the unique solution is

$$
F(x)=\frac{\alpha}{\alpha-\beta}\left[1-\exp \left(\frac{\beta-\alpha}{V}(x-m)\right)\right]
$$

with density

$$
f(x)=\frac{\alpha}{V} \exp \left(\frac{\beta-\alpha}{V}(x-m)\right) .
$$

If $\alpha<0$, this is not a valid density and hence an equilibrium does not exist.

Lemma 16 Suppose $\eta=0, \alpha-\beta \neq 0$, and $\alpha>0$. Then if a symmetric equilibrium exists, $m=0$ and the distribution function is of the form in Eq. (19) with $m=0$.

Proof First, note that since $\eta=0$, there can be no mass point by Lemma 3. Moreover, the solution to the differential equation takes on the form in Eq. (19). Since $\alpha>0$, Lemma 4 implies $m=0$.

Lemma 17 Suppose $\eta=0, \alpha-\beta \neq 0, \alpha>0$ and $\beta<0$. Then there does not exist a symmetric mixed-strategy equilibrium.

Proof By Lemma 16, under this parameter configuration $F(x) \leq \alpha /(\alpha-\beta)<1$ for all $x \geq 0$. Hence, $F$ is not a valid distribution function.

Lemma 18 Suppose $\eta=0, \alpha-\beta \neq 0, \alpha>0$ and $\beta=0$. Then there exists a unique symmetric equilibrium and it is in non-degenerate mixed strategies as characterized in Proposition 2.

Proof First, note that since $\eta=0$, there can be no mass point by Lemma 3. Using Lemma 16 and setting $\beta=0$, yields

$$
F(x)=1-\exp \left(-\frac{\alpha}{V} x\right) \text { on }[0, \infty) .
$$

Since this is an exponential distribution, with mean $V / \alpha$, the expected payoff to a player that bids $w=0$ against $F$ is

$$
\begin{aligned}
E U(0) & =-\gamma-\theta \int_{0}^{\infty} x \mathrm{~d} F(x) \\
& =-\gamma-\theta \frac{V}{\alpha}
\end{aligned}
$$

and hence, $E U(w)=E U(0)$ for all $w \in[0, \infty)$. Thus, a player cannot profitably deviate.

Lemma 19 Suppose $\eta=0, \alpha-\beta \neq 0, \alpha>0$ and $\beta>0$. Then there exists a unique symmetric equilibrium and it is in non-degenerate mixed strategies as characterized in Proposition 2. 
Proof First, note that since $\eta=0$, there can be no mass point by Lemma 3. By Lemma 16 , the distribution must have the form in Eq. (19) with $m=0$. Since $\beta>0$, this is a well-defined distribution function on $\left[0, \frac{V}{\alpha-\beta} \ln \frac{\alpha}{\beta}\right]$ regardless of the sign of $\alpha-\beta$. A player that bids $w=0$ against $F$ earns an expected payoff of

$$
\begin{aligned}
E U(0) & =-\gamma-\theta \int_{0}^{\frac{v+\gamma}{\alpha-\beta} \ln \frac{\alpha}{\beta}} w \frac{\alpha}{v+\gamma} \mathrm{e}^{-\frac{\alpha-\beta}{v+\gamma} w} \mathrm{~d} w \\
& =\frac{\theta v+\delta \gamma}{\theta-\delta}+\beta \frac{\theta}{(\theta-\delta)^{2}}\left[\ln \left(\frac{\alpha}{\beta}\right)\right] V
\end{aligned}
$$

where we have used the fact that $\eta=0$ implies $\alpha-\beta=\delta-\theta$. Since $\beta>0$, a player cannot gain by bidding above the upper bound of the support of $F$.

Lemma 20 Suppose $\eta=0, \alpha-\beta \neq 0$, and $\alpha=0$. Then there does not exist a symmetric equilibrium.

Proof Under the conditions stated, differential equation (3) implies

$$
V f(w)-\beta F(w)=0 .
$$

If $\beta<0$, we have a contradiction, so suppose $\beta>0$. The unique solution to this differential equation is

$$
F(w)=\exp \left(\frac{\beta}{V} w-Q\right)
$$

Since $\eta=0$, Lemma 3 implies there are no mass points. This contradicts the fact that $F(w)>0$ for all $w \in[0, \infty)$. Thus, if $\alpha=0$ and $\eta=0$, there does not exist a non-degenerate symmetric mixed-strategy equilibrium when $\beta>0$ or $\beta<0$.

Case $4: \alpha-\beta=0 ; \eta=0$

Lemma 21 Suppose $\eta=0$ and $\alpha=\beta<0$. Then there does not exist a symmetric equilibrium.

Proof First, note that since $\eta=0$, there can be no mass point by Lemma 3. Under the conditions stated, differential equation (3) implies

$$
V f(w)-\alpha=0
$$

or

$$
f(w)=\frac{\alpha}{V}
$$

But since $\alpha<0$, this is not a well-defined density, a contradiction. 
Lemma 22 Suppose $\eta=0$ and $\alpha=\beta>0$. Then there exists a unique symmetric equilibrium and it is in non-degenerate mixed-strategies as characterized in Proposition 2.

Proof First, note that since $\eta=0$, there can be no mass point by Lemma 3. Under the conditions stated, differential equation (3) implies

$$
V f(w)-\alpha=0
$$

or

$$
f(w)=\frac{\alpha}{V}
$$

Furthermore, since $\alpha>0$, Lemma 4 implies that $m=0$. Hence, the unique solution to the differential equation is

$$
F(x)=\int_{0}^{x} \frac{\alpha}{V} \mathrm{~d} w=\frac{\alpha}{V} x \quad \text { on }\left[0, \frac{V}{\alpha}\right] .
$$

The expected payoff to a player that bids $w=0$ against $F$ is

$$
E U(0)=-\gamma-\theta \frac{V}{2 \alpha}
$$

and hence, $E U(w)=E U(0)$ for all $w \in\left[0, \frac{V}{\alpha}\right]$. Since $\beta>0$, it does not pay to bid above the upper bound of the support, as doing so increases costs but not the probability of winning.

Lemma 23 Suppose $\eta=0, \alpha=\beta=0$, and $\theta=\delta \neq 0$. Then there does not exist a symmetric equilibrium.

Proof First, note that since $\eta=0$, there can be no mass point by Lemma 3. Under the conditions stated, differential equation (3) implies

$$
V f(w)=0
$$

or $f(w)=0$ for all $w$. This is a contradiction.

Taken together, the above lemmas exhaustively describe all mixed-strategy equilibria (and non-existence) as summarized in Proposition 2.

\section{A3. Proof of Proposition 3}

Follows directly from refining the partitions of the parameter space derived in Propositions 1 and 2. 
Open Access This article is distributed under the terms of the Creative Commons Attribution Noncommercial License which permits any noncommercial use, distribution, and reproduction in any medium, provided the original author(s) and source are credited.

\section{References}

Basu, K.: The traveler's dilemma: paradoxes of rationality in game theory. Am Econ Rev 84(2), 391-395 (1994)

Baye, M.R., Kovenock, D.: How to sell a pickup truck-beat-or-pay advertisements as facilitating devices. Int J Ind Organ 12(1), 21-33 (1994)

Baye, M.R., Morgan, J.: The folk theorem for one-shot Bertrand games. Econ Lett 65(1), 59-65 (1999)

Baye, M.R., Kovenock, D., de Vries, C.G.: It takes 2 to Tango-equilibria in a model of sales. Games Econ Behav 4(4), 493-510 (1992)

Baye, M.R., Kovenock, D., de Vries, C.G.: Rigging the lobbying process: an application of the all-pay auction. Am Econ Rev 83(1), 289-294 (1993)

Baye, M.R., Kovenock, D., de Vries, C.G.: The all-pay auction with complete information. Econ Theory 8(2), 291-305 (1996)

Baye, M.R., Kovenock, D., de Vries, C.G.: Comparative analysis of litigation systems: an auction-theoretic approach. Econ J 115(505), 583-601 (2005)

Bertrand, J.: (Review of) Theorie Mathematique de la Richesse Sociale par Leon Walras: Recherches sur les Principes Mathematiques de la Theorie des Richesses par Augustin Cournot. Journal Des Savants 67, 499-508 (1883)

Che, Y.K., Gale, I.L.: Caps on political lobbying. Am Econ Rev 88(3), 643-651 (1998)

Che, Y.K., Gale, I.: Optimal design of research contests. Am Econ Rev 93(3), 646-671 (2003)

Chung, K.L.: A Course in Probability Theory, 2nd edn., Orlando, Academic Press (1974)

Dasgupta, P.: The theory of technological competition. In: Stiglitz, J.E., Mathewson, G.F. (eds.) New Developments in the Analysis of Market Structure, pp. 519-547. Cambridge: MIT Press (1986)

Dasgupta, P., Maskin, E.: The existence of equilibrium in discontinuous economic games. I. Theory. Rev Econ Stud 53, 1-26 (1986)

Dasgupta, S., Tsui, K.: Auctions with cross-shareholdings. Econ Theory 24, 163-194 (2004)

D’Aspremont, C., Jacquemin, A.: Cooperative and noncooperative R- and-D in Duopoly with spillovers. Am Econ Rev 78(5), 1133-1137 (1988)

Engelbrecht-Wiggans, R.: The effect of regret on optimal bidding in auctions. Manag Sci 35(6), 685-692 (1989)

Engelbrecht-Wiggans, R., Katok, E.: Regret in auctions: theory and evidence. Econ Theory 33, 81-101 (2007)

Engers, M., McManus, B.: Chairity Auctions. University of Virginia Working Paper (2004)

Ettinger, D.: Efficiency in auctions with crossholdings. Econ Lett 80, 1-7 (2003)

Fehr, E., Schmidt, K.M.: A theory of fairness, competition, and cooperation. Quart J Econ 114(3), 817-868 (1999)

Filiz-Ozbay, E., Ozbay, E.Y.: Auctions with anticipated regret: theory and experiment. Am Econ Rev 97(4), 1407-1418 (2007)

Fu, Q.: A theory of affirmative action in college admissions. Econ Inq 44(3), 420-428 (2006)

Goeree, J.K., Maasland, E., Onderstal, S., Turner, J.L.: How (Not) to raise money. J Political Econ 113, 897918 (2005)

Groh, C., Moldovanu, B., Sela, A., Sunde, E.: Optimal seedings in elimination tournaments. Econ Theory (2009) (forthcoming)

Hehenkamp, B., Leininger, W., Possajennikov, A.: Evolutionary equilibrium in Tullock contests: spite and overdissipation. Eur J Political Econ 20, 1045-1057 (2004)

Helsey, R.W., Strange, W.C.: A game-theoretic analysis of skyscrapers. J Urban Econ 64, 49-64 (2008)

Hillman, A., Riley, J.: Politically contestable rents and transfers. Econ Politics 1, 17-40 (1989)

Jehiel, P., Moldovanu, B., Stacchetti, E.: How (Not) to sell nuclear weapons. Am Econ Rev 86, 814-829 (1996)

Kaplan, T.R., Luski, I., Wettstein, D.: Innovative activity and sunk cost. Int J Ind Org 21(8), 1111-1133 (2003)

Kittsteiner, T.: Partnerships and double auctions with interdependent valuations. Games Econ Behav 44, 54$76(2003)$ 
Konrad, K.A.: Inverse campaigning. Econ J 114(492), 69-82 (2004)

Kura, T.: Dilemma of the equality: an all-pay contest with individual differences in resource holding potential. J Theor Biol 198(3), 395-404 (1999)

Lin, J.: Price matching in a model of equilibrium price dispersion. South Econ J 55, 57-65 (1988)

Lizzeri, A., Persico, N.: Uniqueness and existence of equilibrium in auctions with a reserve price. Games Econ Behav 30(1), 83-114 (2000)

Maasland, E., Onderstal, S.: Auctions with financial externalities. Econ Theory 32, 551-574 (2007)

Maynard Smith, J.: The theory of games and the evolution of animal conflicts. J Theor Biol 47, 209-221 (1974)

Png, I.P.L., Hirshleifer, D.: Price-discrimination through offers to match price. J Business 60(3), 365-383 (1987)

Riley, J.G., Samuelson, W.S.: Optimal auctions. Am Econ Rev 71, 381-392 (1981)

Rosenthal, R.: A model in which an increase in the number of sellers leads to a higher price. Econometrica 48(6), 1575-1580 (1980)

Sahuguet, N., Persico, N.: Campaign spending regulation in a model of redistributive politics. Econ Theory 28(1), 95-124 (2006)

Schaffer, M.: Evolutionary stable strategies for a finite population and a variable contest size. J Theor Biol 132, 469-478 (1988)

Varian, H.: A model of sales. Am Econ Rev 70, 651-659 (1980)

Vickrey, W.: Counterspeculation, auctions and sealed tenders. J Finance 16, 8-37 (1961)

Zhou, H.: R\&D tournaments with spillovers. Atl Econ J 34, 327-339 (2006) 